Research report

\title{
Focused-attention meditation increases cognitive control during motor sequence performance: Evidence from the N2 cortical evoked potential
}

\author{
Russell W. Chan ${ }^{\mathrm{a}, \mathrm{b}, *}$, Phillip M. Alday ${ }^{\mathrm{e}}$, Lena Zou-Williams ${ }^{\mathrm{a}, \mathrm{d}}$, Kurt Lushington ${ }^{\mathrm{d}}$, \\ Matthias Schlesewsky ${ }^{\mathrm{a}, \mathrm{d}}$, Ina Bornkessel-Schlesewsky ${ }^{\mathrm{a}, \mathrm{d}}$, Maarten A. Immink ${ }^{\mathrm{a}, \mathrm{c}}$ \\ ${ }^{\text {a }}$ Cognitive and Systems Neuroscience Research Hub, University of South Australia, Adelaide, South Australia Australia \\ ${ }^{\mathrm{b}}$ Centre for Cognition and Decision Making, Institute for Cognitive Neuroscience, National Research University Higher School of Economics, Russian Federation \\ ${ }^{\mathrm{c}}$ School of Health Sciences, University of South Australia, Adelaide, South Australia Australia \\ ${ }^{\mathrm{d}}$ School of Psychology, Social Work and Social, University of South Australia, Adelaide, South Australia Australia \\ ${ }^{\mathrm{e}}$ Max Planck Institute for Psycholinguistics, Nijmegen, the Netherlands
}

\section{G R A P H I C A L A B S T R A C T}

Focused-attention meditation training increases cognitive control during motor sequence performance: Evidence from the N2 cortical evoked potential. The left ring represents the cyclical processes common in focused attention meditation (adaptedfromMalinowski,2013). Each process covers the related phenomenological, attentional function and brain network activation. The middle ring represents the cognitive control enhancement that resulted from 21 days of meditation training as reported in the manuscript. The right ring represents how motor sequence learning performance and the N2 potential changes.

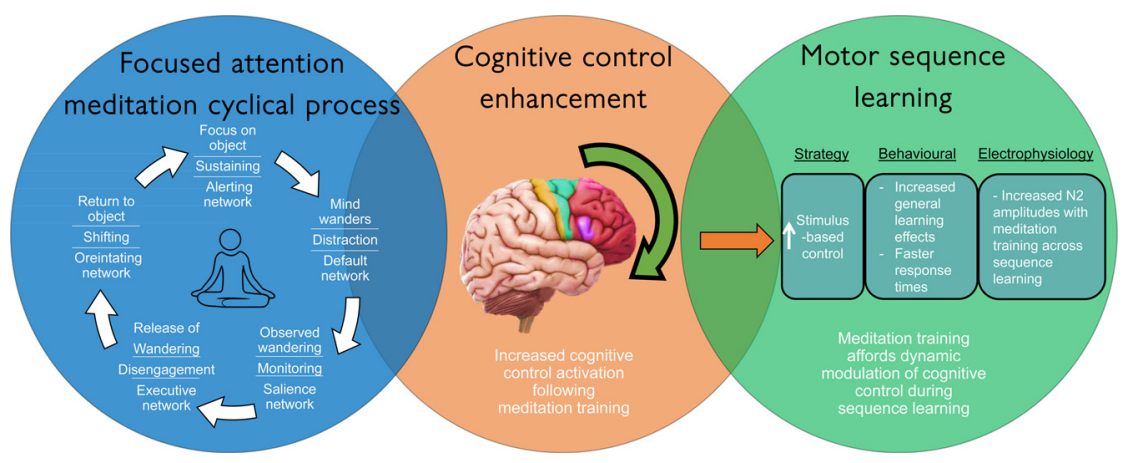

\section{A R T I C L E I N F O}

\section{Keywords:}

Sequence learning

Meditation

Attention

Serial reaction time task

Cognitive control

ERP

N2

\begin{abstract}
A B S T R A C T
Previous work found that single-session focused attention meditation (FAM) enhanced motor sequence learning through increased cognitive control as a mechanistic action, although electrophysiological correlates of sequence learning performance following FAM were not investigated. We measured the persistent frontal N2 event-related potential (ERP) that is closely related to cognitive control processes and its ability to predict behavioural measures. Twenty-nine participants were randomised to one of three conditions reflecting the level of FAM experienced prior to a serial reaction time task (SRTT): 21 sessions of FAM (FAM21, $\mathrm{N}=12$ ), a single FAM session (FAM1, $\mathrm{N}=9$ ) or no preceding FAM control (Control, $\mathrm{N}=8$ ). Continuous 64-channel EEG were recorded during SRTT and N2 amplitudes for correct trials were extracted. Component amplitude, regions of interests, and behavioural outcomes were compared using mixed effects regression models between groups.
\end{abstract}

This research was supported by the Australian Government Research Training Program Scholarship, and the Russian Science Foundation project (RSF-19-1800534) for Russell W. Chan. Ina Bornkessel-Schlesewsky acknowledges the support of an Australian Research Council Future Fellowship (FT160100437).

* Corresponding author at: Centre for Cognition and Decision Making, Institute for Cognitive Neuroscience, National Research University Higher School of Economics, Russian Federation.

E-mail address: russellchanweili@gmail.com (R.W. Chan). 
FAM21 exhibited faster reaction time performances in majority of the learning blocks compared to FAM1 and Control. FAM21 also demonstrated a significantly more pronounced N2 over majority of anterior and central regions of interests during SRTT compared to the other groups. When N2 amplitudes were modelled against general learning performance, FAM21 showed the greatest rate of amplitude decline over anterior and central regions. The combined results suggest that FAM training provided greater cognitive control enhancement for improved general performance, and less pronounced effects for sequence-specific learning performance compared to the other groups. Importantly, FAM training facilitates dynamic modulation of cognitive control: lower levels of general learning performance was supported by greater levels of activation, whilst higher levels of general learning exhibited less activation.

\section{Introduction}

Sequenced actions feature prominently in daily activities of work (e.g. typing, machinery operations) sport (e.g. tennis serve, penalty shoot) and art (e.g. dance, playing a musical score) [1]. Across these activities, a serially-ordered sequence of movements combine to achieve a goal, and the process of attaining them is known as sequence learning [2]. Thus, sequence learning represents a form of goal-oriented behaviour and successful performance requires the interaction of cognitive control processes [3]. Recent theoretical frameworks highlight the importance of cognitive control in sequence learning [4,5]. Specifically, sequence learning aims for optimisation and efficiency [6] and has been shown to be influenced by different cognitive control states previously established by meditation, a cognitive oriented task [3]. Specifically, focused-attention meditation (FAM) has been shown to establish states of increased cognitive control that consequently enhances general response performance but not sequence-specific learning $[3,7]$. The current experiment aimed to further understand the role of cognitive control in sequential behaviour following FAM by investigating a neural correlate of cognitive control, the N2 event-related potential (ERP) [8-11]. In the next sections, we review the theoretical and empirical underpinnings of FAM-induced cognitive control states on sequential behaviour, and the N2 ERP component as an electrophysiological index of cognitive control.

\subsection{Cognitive control in sequential behaviour}

The serial reaction time task (SRTT) paradigm created by Nissen and Bullemer [12] has been widely employed to investigate sequence learning [13-16]. In the SRTT, a stimulus appears at one of several possible locations on a screen and participants respond to the stimuli by pressing a key corresponding to their spatial position. Unbeknownst to participants, the order of the stimuli follows a structured sequence that repeats over several cycles. After several blocks of training, a transfer block with either a new or random sequence is presented, and participants typically exhibit increased reaction times from learning the original sequence in the training blocks. The transfer block introduces a disruption of the original sequence and this allows sequence learning effects to be disentangled from general learning effects, which can be understood by progressive reduction of reaction time in the learning blocks.

Several theoretical frameworks explain the result of cognitive control in sequence representations such as the use of "reaction mode" $[2,16]$ or "stimulus-reflex" [17] during early stages of learning due to low level encoding by forming stimulus-response (S-R) mappings of single targets $[2,13]$. As practice continues, learning allows performance to utilise an associative mode, whereby sequence automatisation slowly increases due to abstract-rule formations [2,18]. Further practice often leads to greater automatisation through "chunking" [5], whereby sequence elements are abstracted into long-term memory representations of smaller sub-units within the overall sequence context $[16,19]$. Chunking allows sequence production to rely less on S-R mappings (since sequence sub-units are preloaded in working memory) and facilitates the anticipation of upcoming responses through greater response-response (R-R) mappings [20,21]. At this stage, performance becomes less stimulus reliant as responses are increasingly driven by an internalised sequence representation. Therefore, when the transfer block in the SRTT is introduced, increased response times are expected due to increased R-R mappings of the original sequence.

Given that sequential tasks represent a form of goal-oriented behaviour, cognitive control is thought to influence sequence learning $[22,23]$ and moderates how a sequential task is represented [5,19,24]. Cognitive control relates to a set of information regulation processes, including attention narrowing and interference inhibition or suppression, that are implemented to ensure successful goal-oriented behaviour $[25,26]$. Cognitive control is also thought to influence how different strategies of sequential behaviour such as stimulus-based control and plan-based control emerge [4,5,27]. Both strategies drive improvements in sequential performance, although through distinct cognitive control processes. For example, in the SRTT there is interference between target and non-target stimuli in the production of each response. To resolve this interference, local competition must be increased based on two cognitive control processes: 1 ) attention narrowing [28,29] and; 2 ) increased inhibition of non-target stimuli or responses [25,30]. Thus, increased cognitive control supports stimulus-based planning and strengthens S-R mapping such that a stimulus is more efficiently translated into a response [2,4,16,17]. Performance improvements associated with stimulus-based control are described as aligning with general learning since the source of the improvement is linked with initial stages and an integral part of sequence learning [31]. General learning effects can be measured in the SRTT, by either the difference in reaction time between the first and last learning block [32,33], or by calculating a coefficient of change in reaction time across all learning blocks $[3,34]$.

Response-based planning requires local competition between responses to be decreased in order to allow formation of a sequence representation, or R-R mapping [4,5]. Thus, response-based planning requires reduced states of cognitive control [22,23] in order: 1 ) to allow working memory to process successive response information [35,36]; and 2) to facilitate response automatisation through predictive behaviour $[37,38]$. As performance is intimately tied to the structure of the sequence representation, any performance gains established by training are lost when this structure is removed. In the SRTT paradigm, the degree by which plan-based control is utilised can be assessed by the degree of sequence-specific performance that is observed based on the lengthening of reaction time between the last learning block involving the sequence and a transfer block, where the sequence is absent or changed [32,33]. Thus, a loss of performance between learning and transfer is indicative of sequence learning.

Cognitive control has the potential to influence sequential behaviour in two distinct ways $[23,39,40]$. Increased cognitive control narrows attention and suppress distractions to allow for speeded responding in a vigilant state [41]. Alternatively, cognitive control weakening affords speeded responding through anticipatory response planning based on the development of sequential representation with tonically active attention [42]. Whether sequential behaviour occurs under states of increased or decreased cognitive control can be determined by a preceding, unrelated goal-oriented task since cognitive 
control states are thought to be inert when shifting between different tasks [40,43,44]. In sequence learning, inducing states of increased cognitive control have been shown to bias stimulus-based control [45], and inducing states of weakened cognitive control may bias plan-based control $[22,46]$ that support learning improvements.

\subsection{Focused-attention meditation strengthens cognitive control in subsequent sequential behaviour}

Recent studies involving focused-attention meditation (FAM) have established evidence of its ability to modulate cognitive control processes resulting in enhanced attention regulation [10,47,48]. The goal in the practice of FAM is to focus attention on an object such as the breath or awareness of body parts [49,50]. It is thought that FAM trains attention regulation by operationalising cognitive control processes required to maintain goal-relevant information and inhibit irrelevant information [25,51]. For example, FAM practice starts by sustaining attention on the object, monitoring and recognising distraction occurrences, and then cognitive control facilitates disengagement from the distraction and shifts attentional focus back to the original object $[10,47,51]$. This ongoing cyclic process of attention regulation entrains cognitive control networks related to alerting, orienting and executive functions in the cortex, and thought to underly neuroanatomical and functional changes from meditation practice (see graphical abstract) [10,52].

Investigation into the influence of FAM states on sequence learning has revealed that when a single-session of FAM immediately precedes learning, enhanced stimulus-based planning contributes to higher rates of response time reductiond [3]. This suggests that the state of increased top-down control established in the FAM state persists to influence subsequent sequential behaviour [7]. However, it appears that strengthened top-down control following FAM is not lasting. Specifically, a delay between the FAM session and sequence learning results in greater reliance on plan-based control, which in turn supports higher levels of sequence-specific learning. Increased reliance on plan-based control suggests that cognitive control is weakened in the delay between FAM and sequence learning [7]. Follow up work provided further evidence that when a single-session of FAM immediately precedes sequence learning, the pattern of learning reflects heightened general learning as opposed to sequence-specific learning [3]. In this work, a session of FAM was compared to completion of a visual attention task, which was equal in duration. Both of these conditions were also contrasted with a quiet-rest condition when evaluating subsequent reaction time performance and sequence learning. Completion of either FAM or the attention task immediately prior to sequence learning resulted in faster and more accurate performance relative to control. However, FAM resulted in greater general learning than the attentiontask. The results were interpreted as illustrating that states of strengthened cognitive control were induced by both FAM and attention task conditions resulting in enhanced performance. However strengthened cognitive control was better able to transfer to the sequence learning following FAM to enhance general learning effects.

An important question that remains unclear in the effects of meditation states on sequence learning is if these effects can be enhanced by meditation training. Further, the neurocognitive mechanisms underlying transfer effects from meditation states to sequence learning have not been delineated. While it is predicted that training may further strengthen cognitive control effects, more experimental evidence is required. The evidence presented thus far has also relied mainly on behavioural measures to infer cognitive control effects. This is a limitation because behavioural measures are often a net outcome of different cognitive processes, making it difficult to understand each relative contribution [53]. This prevents direct inference on the mechanistic actions by which FAM influences sequence learning through cognitive control processes. The acquisition of event-related potentials (ERP) may help to overcome the issue of reliance on behavioural data and thereby strengthen the understanding of key mechanisms by which FAM states and training might contribute to enhancement of sequential behaviour and learning.

\subsection{The N2 event-related potential in sequence learning and meditation}

The use of ERPs provides a more precise approach of estimating changes in neuronal fluctuations involved in cognitive control processes $[53,54]$. The frontocentral N2 component that peaks between 200-450 $\mathrm{ms}$ after stimulus onset is particularly relevant because of its sensitivity to both attention and cognitive control for performance monitoring [55]. Because of its latency, the N2 has been found to be representative of cognitive processes that support sequence learning, such as stimulus orienting [56], stimulus discrimination [57,58] and target selection $[59,60]$. In addition, the anteriorly-distributed N2 was also found to be sensitive to cognitive control, with fluctuations in amplitude considered to index the level of cognitive control regulation [56]. Larger deflections in N2 amplitudes may therefore be indicative of strengthened cognitive control for processing information, while lower amplitudes may be indicative of relatively weakened cognitive control [61].

Earlier ERP studies utilising the SRTT paradigm to investigate the N2 during sequence learning performance aimed to understand contributions from the paradigm of awareness (implicit/explicit) rather than the cognitive control perspective [62-66]. For example, Russeler \& Rosler [65] found that increased N2 amplitudes were evident in learners aware of the sequence for both perceptual and motor deviants, compared to learners that were unaware of the sequence. Extrapolating these findings and reinterpreting them from a cognitive control perspective, an increased N2 amplitude for deviants is indicative of a disruption to the current sequence representation [15]. Increased cognitive control (represented by greater amplitudes) is therefore required to resolve deviances and to maintain equal or greater levels of behavioural performance $[56,67]$. It might also imply that increases in the N2 amplitude (independent of congruency) may serve as a neurobiological signal for increased cognitive control during sequential behaviour.

The amplitude of the N2 component has also been associated with sequence learning. Kobor et al. [68] reported reduced frontocentral N2 amplitude associated with the processing of learned sequenced triplet responses in comparison to random triplet responses. As N2 amplitude is thought to reflect levels of cognitive control employed in goal-oriented behaviour [15,56,69,70], the findings by Kobor et al. [68] further illustrate the relationship between cognitive control and sequential behaviour $[15,68]$. Specifically, heightened N2 amplitudes under random sequential responding reflect heightening of cognitive control to optimise stimulus-based planning, while response-based planning, implemented in the presence of a sequence structure, corresponded with reduced cognitive control, that was observed as reduced N2 amplitudes [4,5,23,68,71].

Let us now focus on how FAM influences the N2 to further predict what effects are plausible for sequence learning. Moore et al. [11] studied the effects of 10-minute daily FAM training over 16 weeks on changes in the $\mathrm{N} 2$ that were associated with the attentional processes in the Stroop task. It was found that meditation training led to a relative increase in N2 amplitudes in both hemispheres (regardless of congruency), although no behavioural differences in Stroop performance were found. One of the methodological gaps of their work was the lack of an attempt to correlate the behavioural results to $\mathrm{N} 2$ amplitudes for further insight into differences between meditators and controls, which we attempt to address in the current experiment. In another study, van Leeuwen, Singer, \& Melloni [72] found that meditators proficient in FAM had more constrained attentional control and performed faster in local targets compared to global targets in the global/local task (indicative of increased cognitive control) than meditators in another style. In addition, FAM meditators were also found to have more negative N2 amplitudes compared to controls during the global/local task 
processing. The current evidence appears to suggest that FAM training will strengthen states of increased cognitive control, reflected by greater N2 amplitudes during cognitive task performance compared to performing other control conditions. There are currently no studies that have investigated the effects of single-session FAM, and brief FAM training on $\mathrm{N} 2$ changes during sequence learning through a randomised design.

\subsection{The present experiment}

Previous work has demonstrated that a single-session of FAM induces states of strengthened cognitive control that promotes stimulusbased control during subsequent motor sequence learning [1,3,7]. In the present experiment, we aimed to replicate the behavioural findings of single-session FAM and, in addition, to understand effects of FAM training (once daily for 20 days) on single-session effects and subsequent sequence learning. Here, we predict that cognitive control effects from additional training would be further enhanced in comparison to single-session FAM but that the mechanistic action of this enhancement may not be easily revealed by behavioural measures. Therefore, we also aimed to further improve inferences on how cognitive control influences the mechanisms underlying sequence learning by recording participants' EEG and examining the N2 component.

Firstly, we predict increased N2 amplitudes during an SRTT for learners that have performed FAM training in comparison to learners engaged in either a single-session of FAM practice or an active-listening control task. Increased N2 amplitudes would reflect enhanced cognitive control effects and will be in line with behavioural performance such as increased general learning performance predicated on stimulus-based control. Motivated by current work to further advance the understanding of electrophysiology and sequence learning effects [68], we wanted to further examine the functional role of the N2 by using regression-based modelling to test and establish links between N2 amplitude and the different behavioural indices of sequence learning.

\section{Methods and material}

\subsection{Participants}

Thirty-three volunteers responded to recruitment from flyers placed in university campuses around Adelaide, Australia and online advertisements on social media. These advertisements targeted 18-35 year old adults living within $25 \mathrm{~km}$ radius of Adelaide. Our exclusion criteria were: participants must not practice any form of meditation regularly in the last year, or taken part in any motor learning experiment in the last year (cross-checked via our experimental database). They must be naturally right-handed; had normal or corrected vision; must be native English speaker or possess native-like English proficiency; be physically healthy without any learning disabilities; and be free from mental health issues and neurological disorders. Typists, piano players, gamers or professionals that engage in high levels of fine finger movements were also excluded. Lastly, they had to agree and be willing to perform the cognitive training task (meditation or listening control) daily, which lasted $22 \mathrm{~min}$ per session, and they had to log their daily practice via an online form.

Of the 33 participants, one participant ceased participation at the second data collection session. Following completion of data collection, three further participants were excluded: one participant revealed that he/she was a regular meditation practitioner in the past after data collection ended; one participant did not practice the mental training correctly; one participant had incorrectly acquired EEG recordings. Data from 29 participants (18 females, $23.1+4.4$ years; $100 \%$ selfreported right-handed) remained for final analysis. The term 'meditation' was not used during the experiment to minimise participation bias. Participants were unaware of specific experimental aims during recruitment but were told that they were participating in an experiment that entailed cognitive training and sequence learning. The research protocol was approved by the University of South Australia Human Research Ethics Committee No. 35297 and all participants provided written informed consent prior to participation. When participants completed all four data collection sessions, they received AU\$100 to compensate for their time and travel cost associated with participation in the experiment.

\subsection{Serial reaction time task (SRTT)}

The stimuli in the current experiment's SRTT involved a row of four hollow boxes that were spatially mapped to four response keys on a modified keyboard numbered 1-4 from left to right. The participants used their index and middle fingers of the left (middle finger on key 1 and index finger on key 2) and right hand (index finger on key 3 and middle finger of key 4) spatially mapped to the numbered keys for responding. The response key was signalled when one of the four boxes was filled in red colour (please see Chan et al. [7] for diagram).

Stimuli were presented using E-Prime ${ }^{\circledR}$ 2.0 Software (Psychology Software Tools Inc., Sharpsburg, USA) on a 21-inch Velta DE-770 BA (Dongguan, China) CRT monitor running at $1680 \times 1050$ pixel resolution in 64-bit colour with a screen refresh setting of $100 \mathrm{~Hz}$. At a sitting distance of $\sim 60 \mathrm{~cm}$ from the display (not strictly enforced), the visual angle dimension of each box was $2^{\circ} \times 2^{\circ}$ with a $2^{\circ}$ separating each box. After each response, a $350 \mathrm{~ms}$ response-stimulus interval (RSI) occurred before the presentation of the stimulus for the next trial. The duration of the RSI was set to ensure that previous trial influences did not affect the ERP recordings between trials, and at the same time this ensured that the SRTT was experienced as a continuous task and not individual key presses. Inaccurate responses were notified with an error message ("Key Error") and all four boxes were marked with an " $\mathrm{X}$ " in the centre with no opportunity to correct their errors before the next trial was presented. At the end of each block, performance feedback was provided in terms of block averaged reaction time (RT) and count of error trials. Following feedback presentation, participants received a 30 $\mathrm{s}$ rest interval. Event markers were recorded at the start of each block (indicating block number), at trial presentation (stimulus), at response initiation (response) and a separate marker for whether the response was a correct or erroneous.

In the Familiarisation session (baseline), participants engaged in the SRTT that was organised into three familiarisation blocks 1-3. Each familiarisation block consisted of 120 pseudorandom stimulus presentation schedules with the condition that stimulus presentation was not repeated on consecutive trials and that each stimulus was presented with equal proportions in each block. A trial represented the presentation of one stimulus and the production of one response. In the final Learning session after the experimental manipulation, participants engaged in the SRTT that was organised into 12 sequence learning blocks. Each sequence learning block comprised of 120 trials per block and a 12-item second order conditional (SOC) sequence (i.e. 121342314324) [73]. Following completion of sequence learning blocks, participants then completed one transfer block (Block 13) involving only pseudorandom trials presented in the same manner as the familiarisation blocks, and a final sequence learning block (Block 14), where the learnt SOC sequence was re-introduced.

\subsection{Focused-attention meditation (FAM) and control task}

Guided by the meditation taxonomy by Nash and Newberg [74], the current experiment operationalised yoga nidra as a cognitive-directed type of FAM that involved four stages: preparation, directing attention to body regions, directing attention to natural breathing and completion [75]. The FAM technique was shown to bias cognitive control in subsequent sequence learning and full transcript was reported in Chan et al. [7]. The FAM was performed in a seated position with eyes closed, with palms turned upwards resting on the thighs for the duration of 22 
min. Instructions were audio-recorded using the voice of a male professional meditation instructor.

Active control conditions for meditation related treatments or interventions ensure rigour in meditation research [76,77]. Accordingly, we included an active control task involving an audio listening task (LT). The goal was to suitably contrast FAM against another cognitive listening task involving a shared stimulus modality. We operationalised a set of instructions that guided the listener to mentally perform steps in the maintenance of a garden. Importantly, the LT also delivered procedural information but did not instruct participants to direct or control their attention like FAM. We chose this control in order to examine if the content and cognitive control processes in FAM were unique compared to attentive listening. The LT instructions were compiled from the audiobook "ABC of Vegetable Gardening", Track 9, Chapter 11, Part A [78] from the Internet Archive https://archive.org (direct link: https:// archive.org/details/abcvegetablegardening_1412_librivox). Track duration was edited to ensure duration equivalence to FAM. Usage of the audio tracks for the purpose of this research was under a Creative Commons license.

In the laboratory, both FAM and LT conditions were played using EPrime $^{\circledast} 2.0$ and stereo Altec Lansing (New York, USA) BXR 1220 audio speakers set at a fixed volume of $40 \mathrm{db}$. All participants across conditions were instructed that the aim was to maintain their attention and mentally follow the audio instructions as best as possible, to remain awake and physically still. For their home practice, participants were asked to practice as closely as possible in the same manner performed in the laboratory, although not strictly enforced. Participants also recorded their daily practice sessions via an online Google form, requiring them to enter comments amounting to no more than 150 characters.

\subsection{Electrophysiological recordings and pre-processing}

EEG was recorded continuously from $64 \mathrm{Ag} / \mathrm{AgCl}$ electrodes (ActiCAP, Brain Products) with a BrainVision actiCHamp Active Electrodes amplifier system (Brain Products GmbH, Gilching, Germany). For monitoring eye movements and blinks, the horizontal and vertical electrooculogram (EOG) was recorded with supra- and infraorbital electrodes on the left eye and two electrodes placed next to the external canthi of the left and right eyes. EEG and EOG were sampled at $500 \mathrm{~Hz}$ and impedances were kept below $5 \mathrm{k} \Omega$ during recording. Two additional electrodes TP8 (left mastoid) and TP9 (right mastoid) were online average to form a single A2 channel. Offline preprocessing of data was performed in MNE-Python v0.15 [79]. EEG data was re-referenced to the average of the left and right mastoids to avoid asymmetrical effects. The data was filtered with a digital zero-phase finite impulse response (FIR) band-pass filter $(0.3-30 \mathrm{~Hz}$, passband edge). Epochs containing eye blinks and artefacts were detected and removed using a peak-to-peak technique; the EOG rejection criterion was $150 \mu \mathrm{V}$. The average artefact-free epochs across participants was $80.0 \%$. The breakdown across groups was: $75.7 \%$ (Control); $79.7 \%$ (FAM1); $84.5 \%$ (FAM21).

\subsection{Procedure}

The experimental procedure is depicted schematically in Fig. 1. During the first Familiarisation session, the experiment was explained to participants and they provided informed consent. Once consent was given, participants were fitted with a BrainVision actiCAP to record the EEG, and they were informed that they would perform three familiarisation blocks of a key-pressing task. Participants received written instructions for the SRTT familiarisation on the computer monitor and were briefed that speed and accuracy were of equal importance. Once ready, participants completed three SRTT Familiarisation blocks (1-3) involving pseudorandom stimulus presentation, which allowed for participants to familiarise responding to the SRTT. This session was representative of participants' baseline behavioural and ERP performance.

The next day, participants were invited back to the laboratory for their Baseline EEG collection session associated with their randomly assigned cognitive training conditions. There were three experimental conditions that determined the daily cognitive training they would engage in. Participants allocated to the FAM21 condition were required to practice FAM once-a-day for the next 19 days as their cognitive training (following session one in the lab), and on the final data collection session, perform a single-session of FAM prior to SRTT learning. Those in the FAM1 condition were required to practice the control LT for the next 19 days as their cognitive training, and on the final data collection session, perform a single-session of FAM prior to SRTT learning. In the Control condition, participants were required to practice the LT for the next 19 days as part of their cognitive training and on the final data collection session, perform a single-session of the LT prior to SRTT learning. This Baseline EEG session was considered each

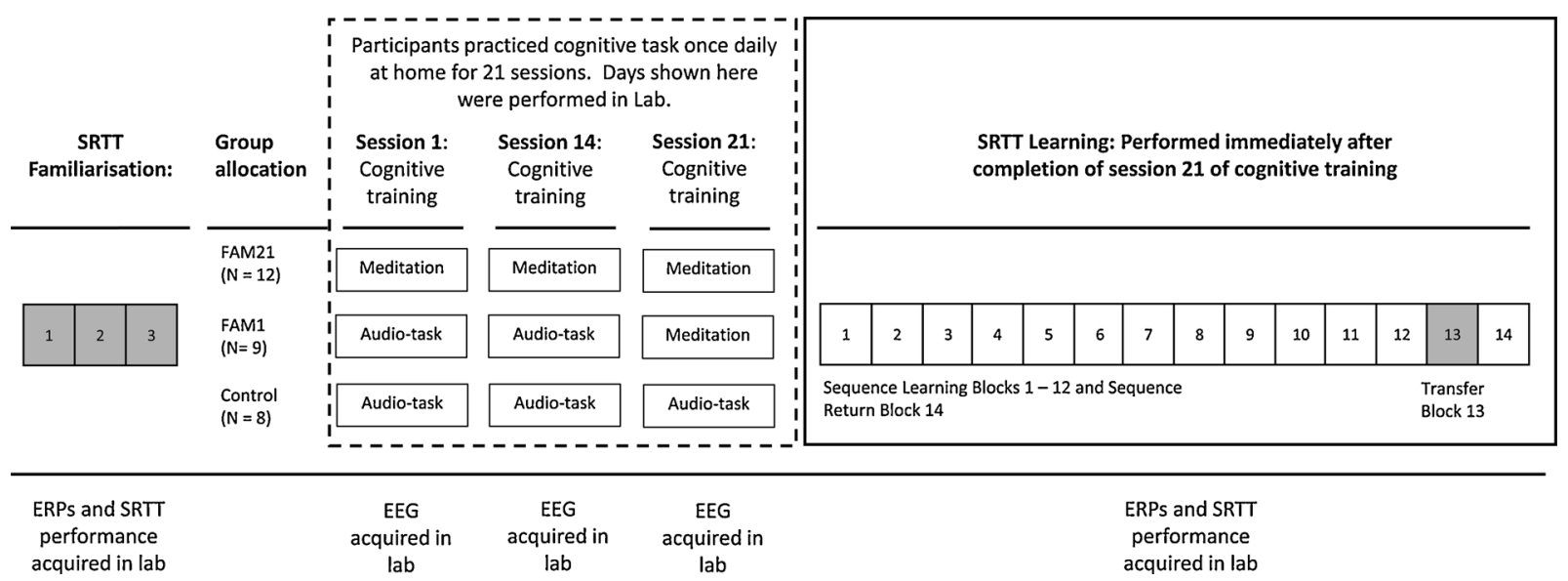

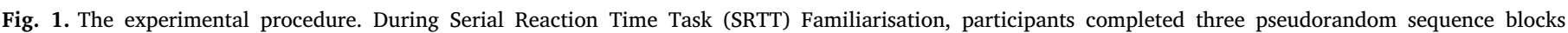

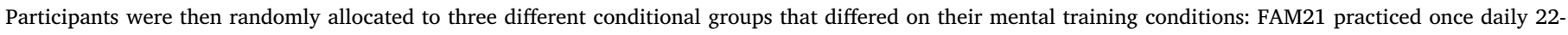

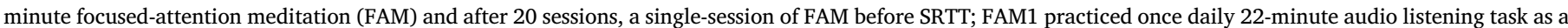

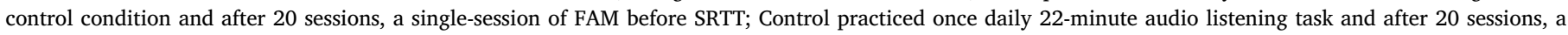

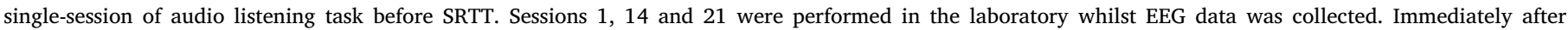

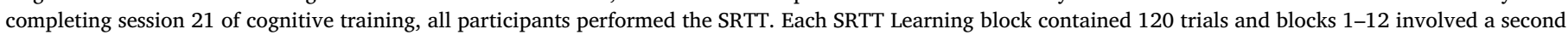

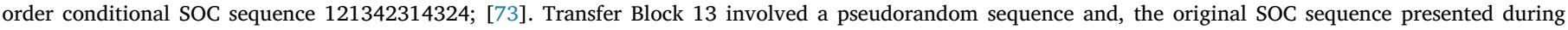
Sequence Learning Blocks was reinstated in the final Block 14. 
participants' first day of cognitive task practice and EEG data was recorded. On day 14 of daily cognitive training, participants returned to the laboratory to perform their cognitive practice whilst EEG was recorded. On days that participants attended the laboratory, they were not required to perform a home practice.

Following completion of 20 days of training in FAM or LT, participants returned to the laboratory for a final EEG recording whilst performing single-session FAM or LT practice (Day 21), followed by sequence learning. The scope of research for the three EEG recorded sessions of FAM were aimed to uncover the differences in oscillatory activity of the different cognitive tasks and will be reported in a separate manuscript.

After completion of the cognitive task EEG recording, we checked equipment and ensured that EEG impedances were still below $5 \mathrm{k} \Omega$ before starting the ERP recordings for SRTT performance. This process took approximately 5-10 min for each participant. Once ready, participants were presented with instructions for the SRTT and again reminded that response speed and accuracy were of equal importance for performance. Participants then completed 12 sequence learning blocks (1-12) where unknown to participants, stimulus presentation followed the repeating 12-item SOC sequence. A transfer block (13) was then presented followed by a final block (14) where the original SOC sequence was re-introduced.

\subsection{Data analysis}

\subsubsection{SRTT reaction time}

Participant RT performance for SRTT Familiarisation and Learning was analysed through linear mixed-effects regression models using the lme4 package Ver. 1.1-21 [80] in the RStudio environment Ver.

\section{A)}

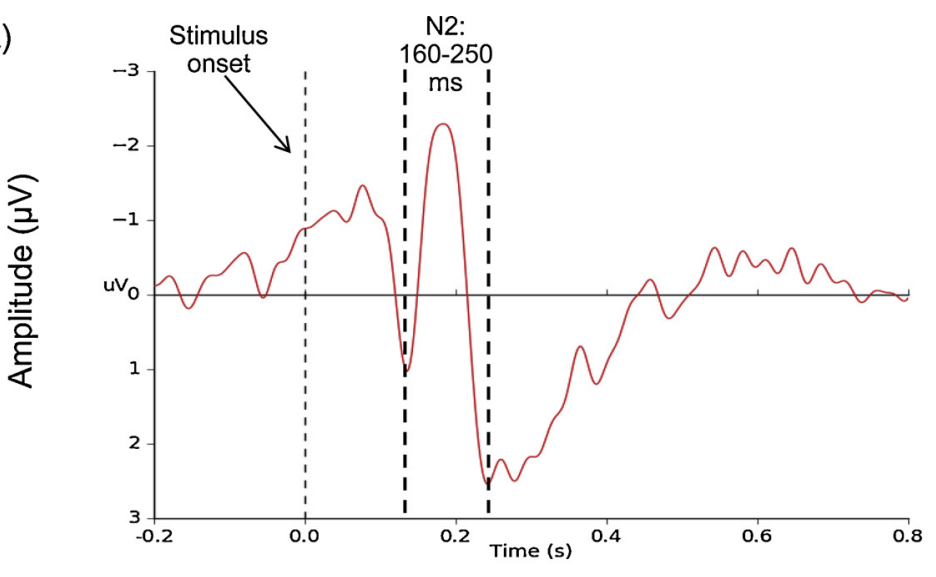

B)
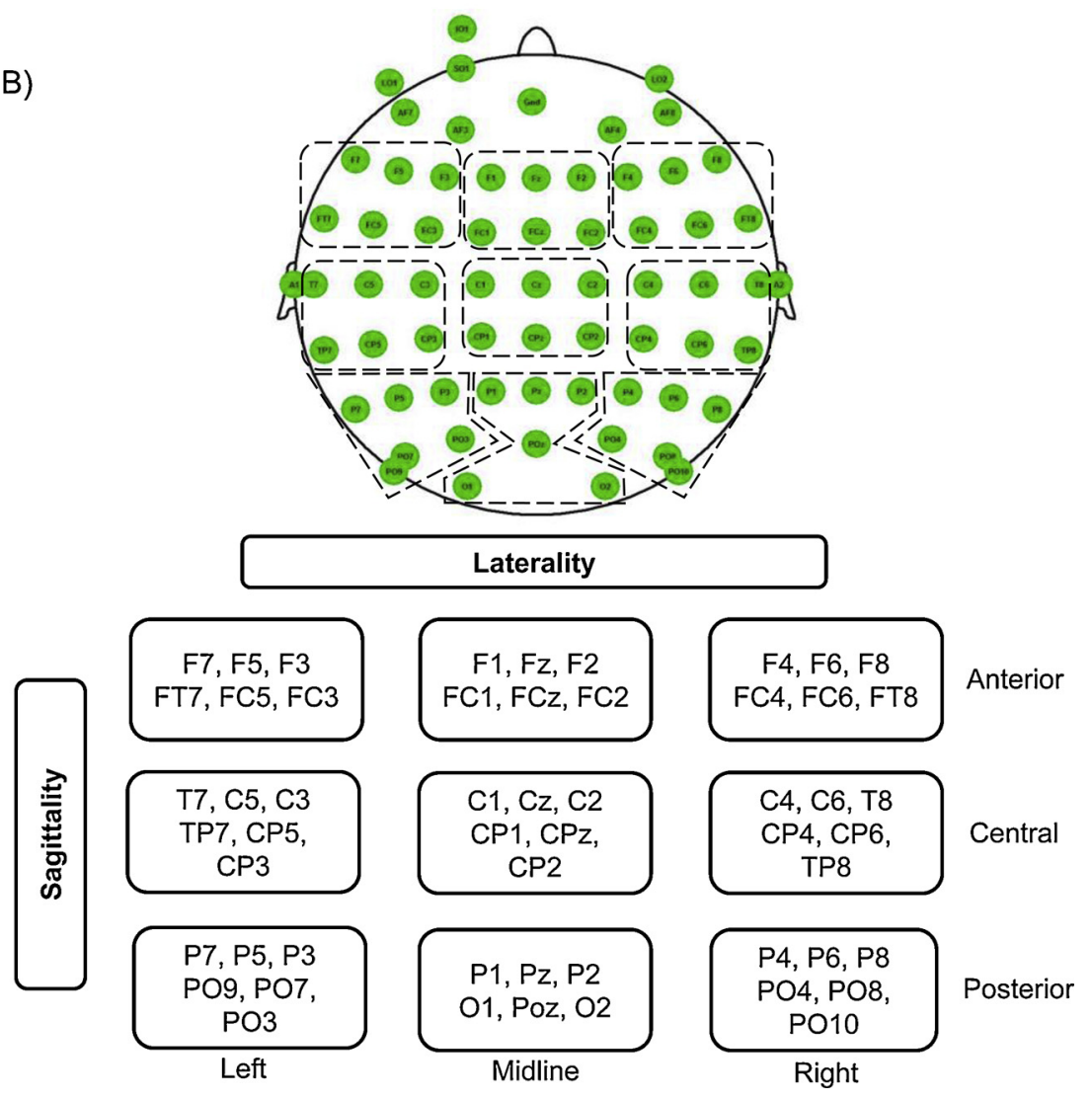

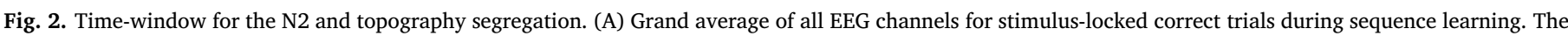

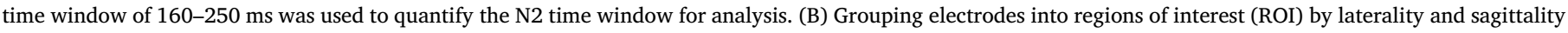
for N2 analysis. 
1.2.5033 [81]. Mixed effects models (MEM) allowed for the control of by-subject differences. In our main model for RT analysis, the outcome variable was trial reaction time (ms) with the predictor variables: (1) Group (i.e. FAM21, FAM1 or Control), (2) Block, (3) Response error (i.e. whether trial was an error or not). Models were constructed separately for Familiarisation blocks (1-3; first laboratory visit) and Sequence learning blocks (1-14; final laboratory visit). We specified subjects as a random factor to control for their associated intraclass correlation (i.e., random intercept models) [82]. Across all MEMs in this experiment (both RT and ERP), we present type II Wald chi square tests with Kenward-Roger degrees of freedom approximation alongside MEM results as this represents the level of confidence in the estimate of effects for each factorial comparison. Finally, to determine the locus of block level interaction, we estimated the marginal means of group performance using emmeans package Ver. 1.4.3.01 [83] in the $\mathrm{R}$ environment.

2.6.2. Model-based approach for sequence learning indices as predictors for amplitude in ERP components

For the current experiment, in addition to understanding group differences of motor sequence learning indices, we used a model-based approach to test each of the learning indices and their relationship with the N2 component. This approach would entail calculating each of the learning indices and then using them as predictors in the statistical models. Our theory-based prediction is that the general learning index would provide the best model fit for the N2 component. The rationale based on the theoretical frameworks is that general learning performance improvements are associated with stimulus-based control under the notion of increased cognitive control [31].

The investigation of motor sequence learning performance indices in the form of general learning performance and sequence-specific learning provides an important dimension towards the understanding of electrophysiology and behavioural performances changes. To establish an index of general learning performance, we computed each participant's rate of performance improvement (i.e., reduction in RT) across SRTT Learning Blocks 1-12, when the SOC sequence was presented. A separate regression analysis of RTs (correct-only trials) as the independent variable and trial number as a dependent variable guided by a methodology established in Verstynen et al. [34] was performed. However, instead of determining asymptomatic learning by choosing either linear or quadratic models, we summated the beta coefficients for each participant's linear and quadratic model to simplify model selection. The goal was to allow for a more unbiased approach across all participants when we modelled their learning rates against N2 amplitudes. The beta coefficient was considered the degree of trial RT change during learning, representing general learning effects during SRTT performance [7]. Greater negative values represented better general learning performance and were interpreted as reflecting increased stimulus-based control $[3,7]$.

The sequence-specific learning percentage was computed based on mean reaction time (MRT) performance between blocks 12 and 13. It was calculated by subtracting 1 from the ratio of block 13 MRT relative to block 12 MRT and then multiplying by 100 . Increasing percentage values were indicative of greater levels of sequence-specific learning. We have previously found that the sequence-specific learning percentage was representative of plan-based control [3,7].

\subsubsection{The $N 2$ event-related potential: data preparation and analysis}

For the N2, the EEG was time-locked to the onset of each trial presentation to create $1000 \mathrm{~ms}$ (ms) epochs consisting of a $-200 \mathrm{~ms}$ pre-stimulus to $800 \mathrm{~ms}$ post-stimulus time window. Individual epochs were then averaged to obtain ERPs. Only the ERPs for correct trials for Familiarisation and Sequence Learning Blocks were analysed. We created the grand average waveform of all correct trial epochs from Sequence Learning Blocks across all EEG channels for the 29 participants to determine the N2 time window. Visual inspection of the grand average aligned with previous research of FAM meditation effects for cognitive control [11], with maximal amplitude in the time window of 160-240 ms which was used to quantify the N2 (See Fig. 2A). Electrodes and their averaged amplitudes were grouped into the nine

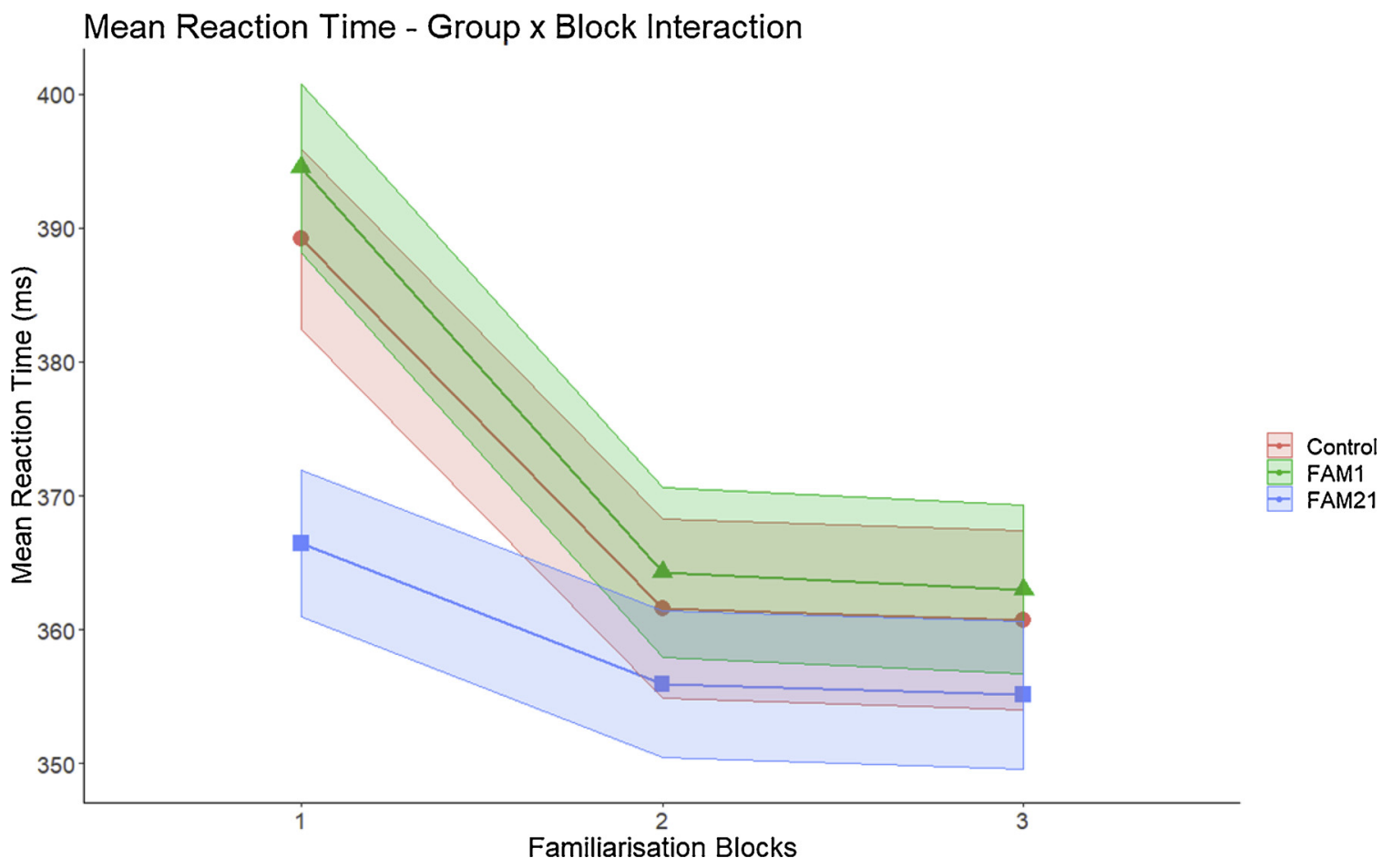

Fig. 3. Familiarisation Mean Reaction Time - Group x Block Interaction. Modelled effects in Familiarisation Blocks 1-3 of the serial reaction time task (SRTT) involving pseudo-random ordered trials. Familiarisation was performed prior to experimental manipulation. RT differences in block 1 between the groups was evident but there was a convergence of performance in blocks 2 and 3, showing equal performance. Error bars represent $95 \%$ confidence intervals. 
regions of interest (ROI) shown in Fig. 2B, to analyse amplitude differences based on scalp topography. This was to further understand regional effects rather than single electrode investigations that are typically reported $[11,68,84]$.

We analysed N2 amplitudes with MEMs using the abovementioned lme4 package in the $\mathrm{R}$ environment. Models were constructed separately for Familiarisation and Sequence Learning related N2. For the Familiarisation N2 model, the outcome variable was correct trial epoch amplitude $(\mu \mathrm{V})$ and the predictor variables were: (1) Group (i.e. FAM21, FAM1 or Control), (2) Laterality, (3) Sagittality. In addition, for the Sequence Learning N2 model, general learning and sequence-specific learning indices were entered into the model separately as a predictor, thus allowing us to quantify the relationship between $\mathrm{N} 2$ amplitude and the two learning indices. Based on Akaike's Information Criterion (AIC) [85] estimates, the index that provided the best fit will be reported. We estimated interaction effects in MEMs using Effects Ver. 4.1-4 [86], whereby learning indices values were binned to estimate the linear effects. Models further included random intercepts by subject to control for their associated intraclass correlation.

We chose to analyse Familiarisation and Sequence Learning sessions separately motivated by two main reasons. Firstly, stimulus structure greatly affects cognitive control in sequence learning and hence considering these two phases as a factor for comparison would be an inequivalent approach due to differences in stimuli presentations between the sessions. Second, the number of blocks were also different and as such, total trial numbers between Familiarisation and Sequence Learning were also unequal, which would affect ERP analysis and inferences. We will address further improvements for future work of ERP and sequence learning in the limitations section.

\section{Results}

\subsection{SRTT reaction time performance: Familiarisation}

Firstly, the MEM analysis of Familiarisation (blocks 1-3) performance revealed that there was neither a significant Group x Accuracy interaction for trial RT $(p=.20)$, nor a significant Group $\mathrm{x}$ Accuracy $\mathrm{x}$ Block interaction for trial RT $(p=.50)$. Accordingly, the model was simplified by removing all incorrect trials [33]. This resulted in the exclusion of $2.6 \%$ of all SRTT trials in Familiarisation from further analysis.

The main Familiarisation MEM model with only correct trials revealed no significant main effect of Group $(p=.65)$ but a significant effect of Block, $\chi^{2}(2, N=29)=112.1, p<.001$, reflecting shorter RT across familiarisation blocks, particularly from the first to second blocks. In addition, a significant Group $x$ Block interaction, $\chi^{2}(4, N=$ $29)=20.1, p<.001$, showed that the relationship between blocks and trial RT changed as a function of Group. Post-hoc Tukey tests revealed that the interaction resulted from differences during block 1 of Familiarisation, during which the FAM21 group showed faster RTs than the FAM1 $(p<0.05)$ and Control $(p<0.05)$ groups. Subsequent blocks 2 and 3 showed that trial RT performance was equal amongst the groups $(p>0.05)$ and converged (see Fig. 3).

\subsection{SRTT reaction time performance: Sequence Learning}

The MEM analysis of Sequence Learning (blocks 1-14) performance revealed that there was no significant Group x Accuracy interaction for trial RT ( $p=.93)$, nor was there a significant Group $\mathrm{x}$ Accuracy $\mathrm{x}$ Block interaction for trial RT $(p=.06)$. The mixed effects model was thus simplified by removing all erroneous trials and Accuracy as a factor for further analysis of RT performance. This resulted in the exclusion of 3.4 $\%$ of all trials in Sequence Learning Blocks.

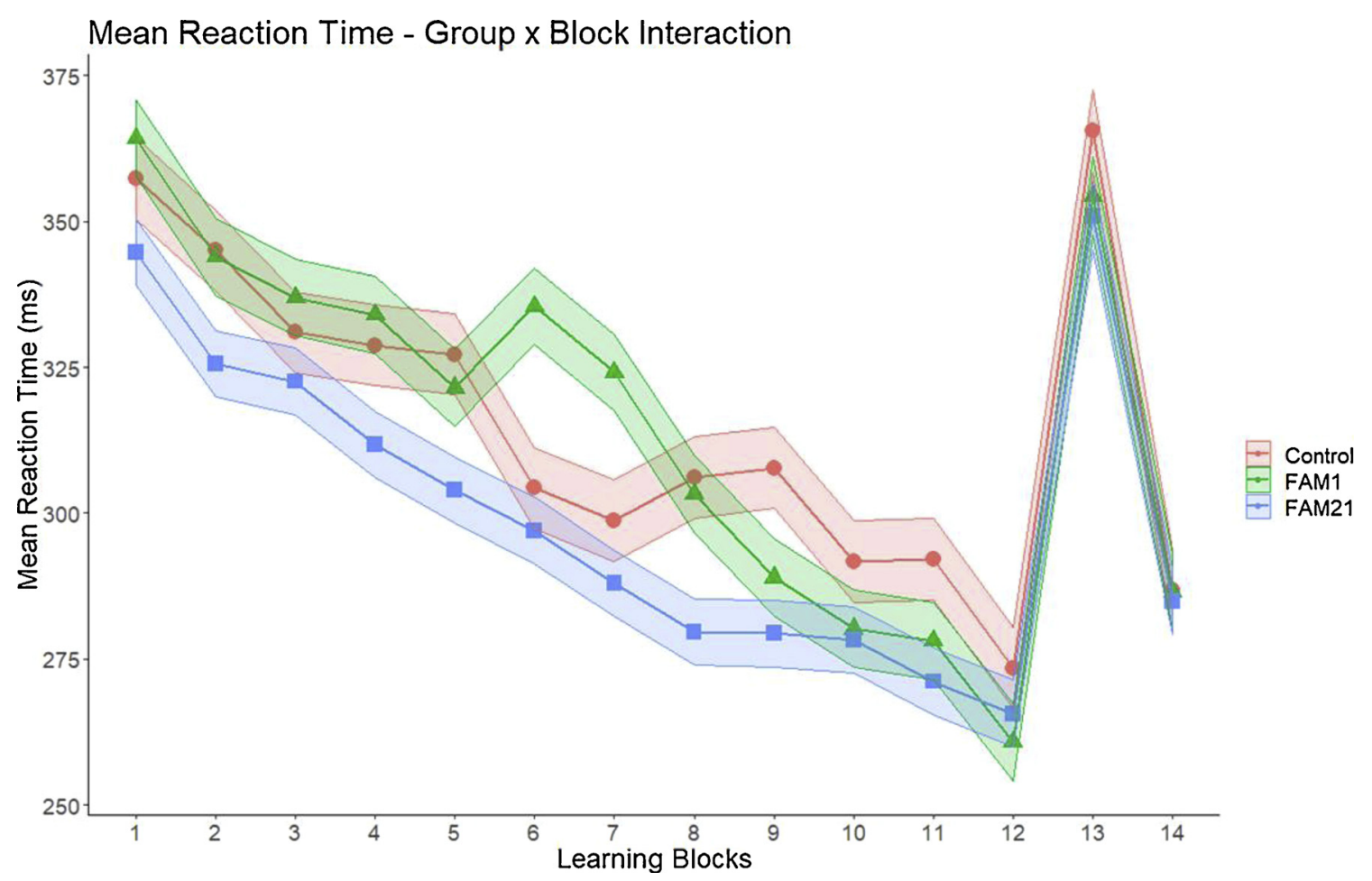

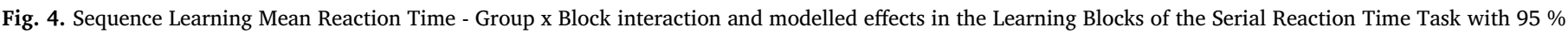

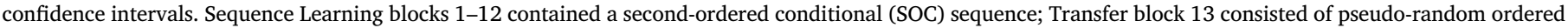

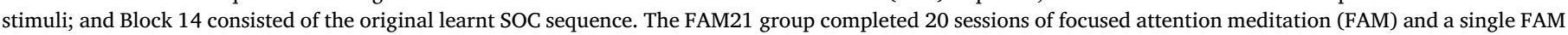

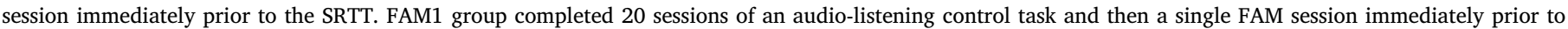

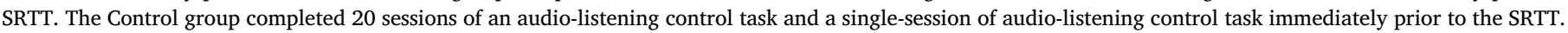


The Sequence Learning MEM for the remaining correct trials revealed no significant main effect of Group $(p=.79)$ but a significant effect of Block on trial RT, $\chi^{2}(13, N=29)=3991.9, p<.001$, where all groups exhibited RT reductions across Learning Blocks 1-12, an increase in RT for Transfer Block 13 and then a reduction in RT for Block 14, which was typical of SRTT performance [32,87]. There was a significant Group $\mathrm{x}$ Block interaction, $\chi^{2}(26, \mathrm{~N}=26)=234.9$, $p<.001$, such that the groups demonstrated differences in RT performance across blocks. Due to the large number of interactions between the blocks and groups, we summarise the results by describing similar effects based on the group comparisons with post-hoc Tukey tests. Overall, the FAM21 group demonstrated shortest RT performance in 11 of 14 blocks, FAM1 only in one block and Control for none of the blocks. Specifically, post-hoc comparisons of the effects showed that FAM21 had shorter RTs compared to Control for 8 of 14 blocks (2, 4, 5, $8,9,10,11$ and $13 ; p<0.05)$, and had shorter RTs compared to FAM1 on 8 of 14 blocks $(1,2,3,4,5,6,7$ and $8 ; p<0.05)$. FAM1 had shorter RTs than Control in 2 of 14 blocks ( 9 and $11 ; p<0.05$ ). Control had shorter RTs than FAM1 in 2 of the 14 blocks ( 6 and $7 ; p<0.05)$. Neither FAM1 nor Control had shorter RTs compared to FAM21 in any of the Sequence Learning blocks. See Fig. 4 for an overview of RT performance in the Learning Blocks.

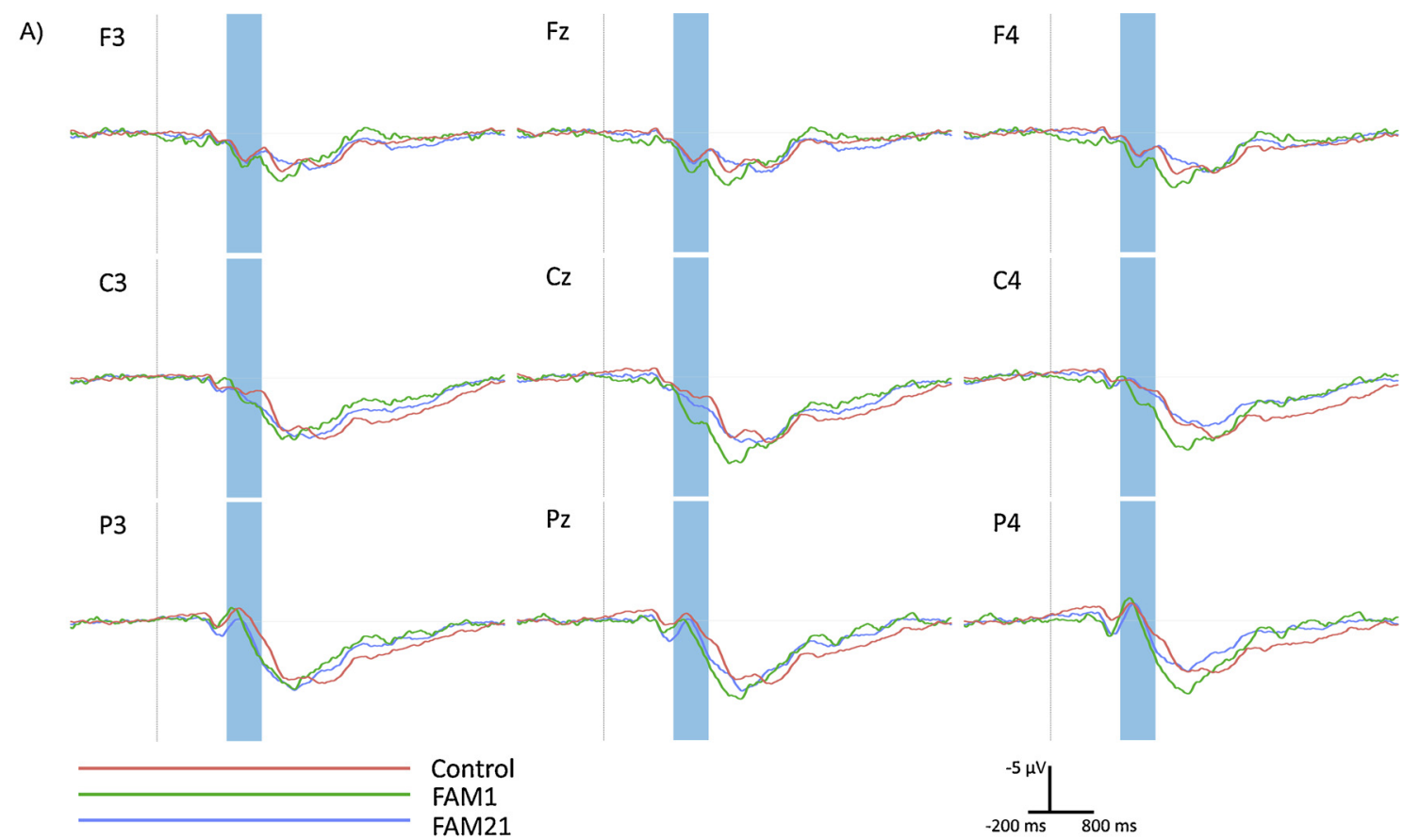

B) Familiarisation N2 Amplitude
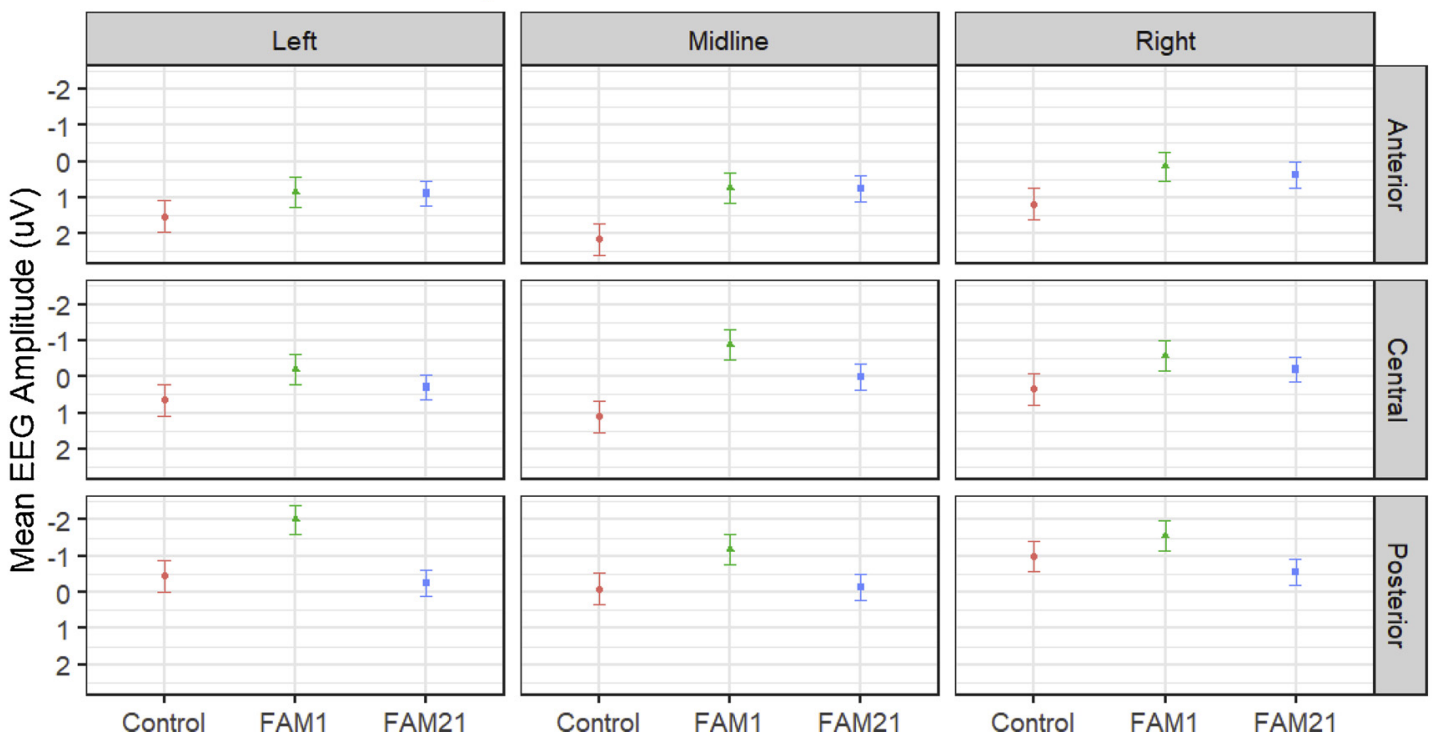

Fig. 5. A) The grand-average plots reflect selected electrodes that are representative for the regions of interest in the statistical model. The N2 component is highlighted by the blue band, for the time range of $160-240 \mathrm{~ms}$ and stimulus-locked to SRTT trial onset for all correct trials performed across Familiarisation blocks. Although groups are indicated, Familiarisation session of the sequence learning task was performed prior to experimental manipulation. B) Modelled effects for Familiarisation N2 Amplitude: Group x Laterality x Sagittality. Error bars represent $95 \%$ confidence intervals, they are very small due to high consistency from correct responses. 


\subsection{N2 Familiarisation}

As no significant Group x Accuracy interactions were evident in either the Familiarisation or the sequence learning phases in the behavioural data, we analysed the amplitudes of only correct trial epochs for the N2. The results of the Familiarisation MEM for N2 amplitude revealed no significant main effect of Group ( $p=.17)$, a significant Group $\mathrm{x}$ Laterality interaction, $\chi^{2}(4, \mathrm{~N}=29)=291.0, p<.001$, a significant Group x Sagittality interaction, $\chi^{2}(4, N=29)=1072.6$, $p<.001$, and a significant Group $\mathrm{x}$ Laterality $\mathrm{x}$ Sagittality interaction, $\chi^{2}(8, N=29)=308.9, p<.001$. In all the anterior (left, midline and right) ROIs, FAM21 and FAM1 both showed increased negativities compared to Control but did not differ from each other. In the centralmidline, FAM1 had increased negativity compared to FAM21 and Control. In the central-right, FAM1 had increased negativity compared to Control but did not differ from FAM21. In the posterior (left and midline) ROIs, FAM1 had increased negativities compared to FAM21 and Control. Both FAM21 and Control in posterior (left and midline) performed similarly. In the posterior-right ROI, FAM1 had increased negativity compared to FAM21, but FAM1 was not different from Control. See Fig. 5B for Group and interaction effects across the ROIs plot. In summary, although differences were evident across ROIs, no main effect of Group was evident, which appears to corroborate with the behavioural results.

\subsection{N2 Sequence Learning: General learning and Sequence-specific learning models}

The model-based approach for determining the sequence index as a predictor for $\mathrm{N} 2$ amplitude revealed that general learning performance provided the better model fit (AIC $=11730322$ ) compared to sequencespecific learning (AIC $=11735120$ ). Therefore, we retained general learning performance as the main factor for the Sequence Learning MEM but also report the N2 Sequence-specific model as way to understand sequence-specific performance and differences. The main and interaction effects are reported in both Table 1 and 2.

We examined the significant Group $\mathrm{x}$ Laterality $\mathrm{x}$ Sagittality (General learning) interaction first as a way to understand the differences in Sequence Learning from Familiarisation (see Fig. 6 for modelled effects). In the anterior (left, midline and right) ROIs, mean amplitude between the groups appeared in a gradient manner. Increased negativity was observed in FAM21 compared to FAM1 and Control. FAM1 had greater negativities in the anterior-midline compared to Control only, but not in the anterior-left or -right. In the central-left ROI, FAM21 had greater negativities compared to Control but was not different from FAM1; FAM1 was not different from FAM21 and Control. In the central-midline ROI, FAM21 had increased N2 negativity compared to FAM1 and Control; FAM1 N2 was more negative compared to Control. In the central-right ROI, only FAM21 had increased N2 negativity compared to Control; FAM1 was not different from Control. In the posterior-left, both FAM21 and FAM1 had the greatest negativity and were different from Control. In the posterior-midline, FAM21 had the greatest N2 negativity and was different from Control but not FAM1. Lastly, in the posterior-right ROI, all three groups had similar N2 negativity.

We reported the Group x Laterality x Sagittality x General learning interaction to understand the effects between cognitive training, general learning performance and N2 amplitude in the different regions. The pattern that emerged revealed that for FAM21 participants, at lower levels of general learning performance $(0-1.5)$, greater N2 negativities were evident compared to other groups to maintain the same levels of general learning performance compared to FAM1 and Control. However, as general learning performance improves (1.5- > 3), FAM21 had the greatest rate of reduction in N2 amplitude compared to FAM1 and Control. At the highest levels of general learning performance, the most pronounced effects in FAM21 compared to FAM1 and Control were observed in the anterior (left and midline) and central (left and midline) ROIs. In the anterior-left ROI, significantly less negative amplitudes can be expected at high levels of general learning performance compared to FAM1 and Control (Fig. 7).

We reported the Group x Laterality x Sagittality x Sequence-specific interaction to understand the effects between cognitive training, sequence-specific performance and $\mathrm{N} 2$ amplitude in the different regions. The pattern that emerged revealed that for FAM21 participants, at lower levels of sequence-specific learning performance (0-1.5), similar levels of N2 amplitudes are evident across the groups except for the anterior-midline and central-midline ROIs. For the two identified ROIs, increased N2 negativities can be expected from FAM21 compared to other groups to maintain the same levels of sequence-specific learning compared to FAM1 and Control.

As sequence-specific learning performance further improves (1.5- > 3), all groups reduce in their N2 amplitudes in the anterior and central regions. FAM21 maintained higher N2 negativities compared to FAM1 but not Control in the anterior regions. In the central-right ROI, FAM21 was more negative that Control but not FAM1.

In the posterior ROI, a pattern emerged where FAM21 maintained a relatively flat trajectory and $\mathrm{N} 2$ amplitudes, compared to FAM1 that had increasing N2 amplitudes and Control that had a reduction of N2 amplitudes (Fig. 8).

\section{Discussion}

The central thesis of the present work is that cognitive control states established by goal-oriented tasks influence the implementation of executive control strategies [4] in subsequent sequence learning tasks [22]. With respect to FAM, a state of strengthened cognitive control results in the implementation of the stimulus-based control strategy in a subsequent sequence learning task [1,3,7]. We predicted that increased cognitive control that transfers from FAM to sequence learning, would be reflected by elevated N2 amplitudes during performance in the SRTT relative to control conditions [11,54-56,88]. Larger N2 amplitudes during SRTT performance were predicted for those who received preceding training with FAM as compared to those who only experienced a single-session of FAM or a listening control task prior to the SRTT.

The behavioural results showed that FAM training resulted in greater general learning during the sequence learning task as compared to the other conditions. This suggests that training with FAM further enhanced the capacity to establish states of strengthened cognitive control, which transfered to subsequent sequence learning to reinforce stimulus-based control forms of sequential behaviour. Performing a single-session of FAM and listening control resulted in equal behavioural performance, replicating previous work [3]. In terms of the N2 ERP component, increased amplitudes were found in both the FAM training and single-session FAM group compared to the listening control group, suggestive of increased cognitive control during sequence learning. We will begin by further discussing the effects on sequence

Table 1

Overall Sequence Learning main and interaction effects on mean EEG amplitude for the N2 component in correct trials. General learning performance provided the best model fit and was retained as the main predictor for N2 amplitudes.

\begin{tabular}{lll}
\hline Effect & df & $\mathrm{X}^{2}$ \\
\hline Group & 2 & 14.96 \\
Group x General learning & 2 & 10.56 \\
Group x Sagittality x Laterality & 8 & 910.05 \\
Group x Sagittality x Laterality x General learning & 8 & 267.77 \\
\hline
\end{tabular}

Note:

$* p<.05$.

$* * \mathrm{p}<.01$.

$* * * \mathrm{p}<.001$.

NS $=$ Not Significant. 
Table 2

Overall main and interaction effects during Sequence Learning on mean EEG amplitude for the N2 component of correct trials. Sequence-specific performance results when modelled as a secondary predictor for N2 amplitudes.

\begin{tabular}{llll}
\hline Effect & df & $\mathrm{X}^{2}$ & Significance \\
\hline Group & 2 & 12.10 & \\
Group x Sequence-specific learning & 2 & 0.15 & NS \\
Group x Sagittality x Laterality & 8 & 968.56 & \\
Group x Sagittality x Laterality x Sequence-specific & 8 & 95.98 & \\
$\quad$ learning & & & \\
\hline
\end{tabular}

Note:

$* p<.05$

$* * p<.01$.

$* * * \mathrm{p}<.001$.

NS $=$ Not Significant.

learning performance, before turning to the effects on N2 amplitude, and finally, shedding some light on the degree by which N2 amplitude, general learning and sequence-specific performances changes are affected by FAM.

\subsection{Meditation effects on sequence learning performance}

In comparison to the control condition, a single-session of FAM did not show consistent RT improvements during sequence learning. Singlesession FAM was only faster in two blocks and vice versa for the Control. In regard to the comparison between single-session FAM and Control, the current results were in agreement with our previous work [3]. Previous results suggest that performing other cognitive tasks (such as a computerised attention control task or the current listening control task) may also actively engage cognitive control to induce states of increased cognitive control, that carry forward to subsequent sequence learning.

We found that FAM training led to faster performance during sequence learning compared to single-session FAM and Control. Specifically, FAM training led to the shortest RT durations in the majority of all the learning blocks compared to the other conditions. These performance enhancements were associated with general learning as opposed to sequence-specific learning due to the lack of significant perfomance differences between groups when comparing transfer and final learning blocks. This indicates that, on a behavioural level, sequence-specific learning effects between the groups were equal and there were no differences in plan-based control (Table 2) [4,7]. It should be emphasised that enhanced general learning effects from FAM training were evident when performance was compared between groups in the initial blocks of the SRTT. This early performance advantage suggests that FAM training affords increased levels of cognitive control from the onset of sequence learning, resulting in general learning [43]. Neither single-session FAM nor Control demonstrated shorter block RTs compared to FAM training during sequence learning.

These results provide the first behavioural evidence that brief FAM training results in the reinforcement of single-session FAM benefits for general learning performance during sequence learning. On a behavioural level, greater general learning effects are suggestive of increased stimulus-based control as a dominant strategy to resolve local competition between possible targets during trial presentation [4,43]. The performance effects demonstrated in the single-session FAM and performing an equivalent listening control task was also consistent with previous work $[1,3,7]$. Therefore, behavioural results were consistent with our prediction that increased FAM training results in enhances general learning performances during sequence learning but not sequence-specific learning. With the EEG component of our study, we aimed to further investigate if electrophysiological correlates of sequence learning also supported this interpretation.

\subsection{Focused-attention meditation effects on N2 and cognitive control during sequence learning}

Grand mean N2 amplitude during Familiarisation did not show any main group-based differences, which was in line with the behavioural results for overall RT and our expectations. Since at this time, participants did not participate in any cognitive tasks, we expected that participants across groups would be performing similarly to each other. However, despite our expectations we showed that FAM1 in averaged electrodes across the central-midline and posterior-left ROIs had greater N2 amplitudes compared to the other groups. When clarified by representative electrodes grand averages (Fig. 5A), it would appear that groups were performing similarly with accordance to our expectations. Traditionally, the Familiarisation session was created to serve the purpose of allowing participants to orientate themselves to the SRTT task $[12,73]$. Our interpretation is that participants were affected by the pseudo-random sequence differently with FAM1 showing increased amplitudes and therefore suggestive of increased cognitive control to complete the Familiarisation session.

Moving onto the sequence learning results, a previous session of FAM resulted in higher N2 amplitudes during SRTT performance compared to control conditions. Those who received FAM training exhibited further N2 amplitude increases relative to those receiving no training involving meditation. Specifically, a clear pattern emerged from our results across anterior and central ROIs whereby FAM21 exhibited the highest negative amplitudes for the N2 compared to FAM1 and Control. This pattern of activation across the ROIs for the N2 appeared to follow a gradient, suggestive of modulation by the volume of FAM training: the least amount of negativity was exhibited by the Control; greater negativities were found in FAM1; and FAM21 exhibiting greatest levels of negativities for the N2 during Sequence Learning. Firstly, the N2 results are in line with previous work [62,68], which suggests that the N2 is indeed sensitive to cognitive control during sequence learning when interpreted in conjunction with behavioural performance. Specifically, it was found that the N2 amplitudes were smaller in blocks involving sequenced structure compared to the transfer blocks involving randomised patterns, indicating that further strengthening of cognitive control was needed under random presentation conditions [68]. This can be extended to suggest that the larger N2 amplitudes texhibited by the FAM training group reflect greater levels of cognitive control necessary to sustain implementation of the stimulus-based control strategy even when sequence structures where present during learning blocks. However, the difference, as compared to Kobor et al. [68], was that the FAM training group continued to elicit the shortest reaction times across sequence learning blocks rather slowing reactions times. This notion is clarified in the model-based approach of the next section.

The N2 results were also consistent with Moore et al. [11], who investigated the effects of FAM training in a longitudinal (16-weeks) study design utilising the Stroop task. In terms of ROIs, the authors found an increase in N2 amplitudes in posterior channels for meditators compared wait-listed control subjects, attributing to contributions from occipitotemporal areas. The authors found that meditation training resulted in more efficient perceptual discrimination of stimuli because of increased focused attentional resources. Perceptual discrimination is considered only one of the several cognitive control processes that support visual attention to discern between targets and non-targets competing at a local level for the Stroop task $[8,89,90]$. As the Stroop task does not contain a sequence that could be learnt for optimisation, task structure may have partly contributed to the reason why our posterior channels did not show the same interaction effects as the frontocentral channels.

Instead, our results of increased N2 negativities with the abovementioned gradient fashion, were topographically situated in the anterior and central ROIs. Using source estimation techniques, Gruendler et al. [91] found that the frontocentral N2 component had 


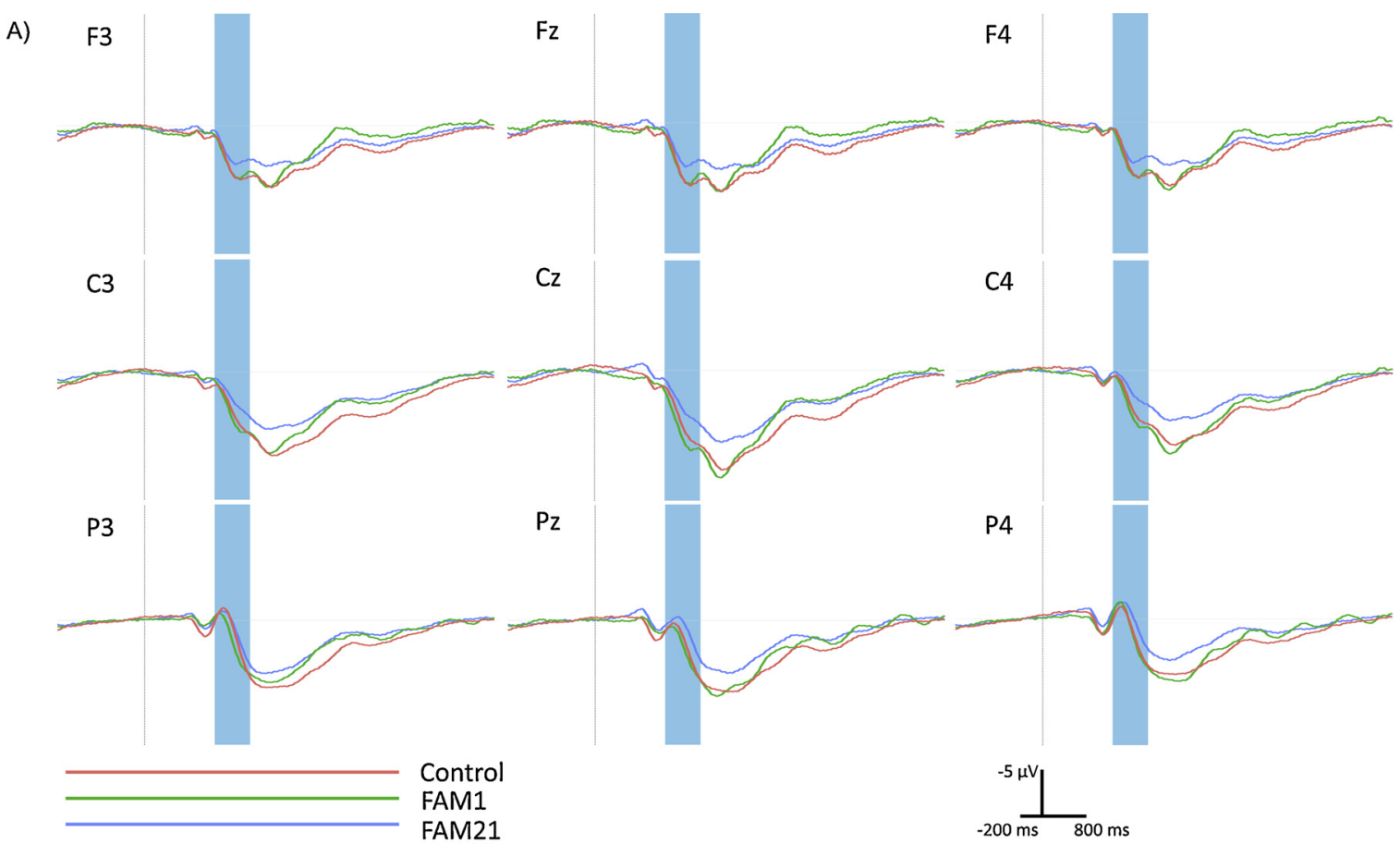

B) Learning N2 Amplitude
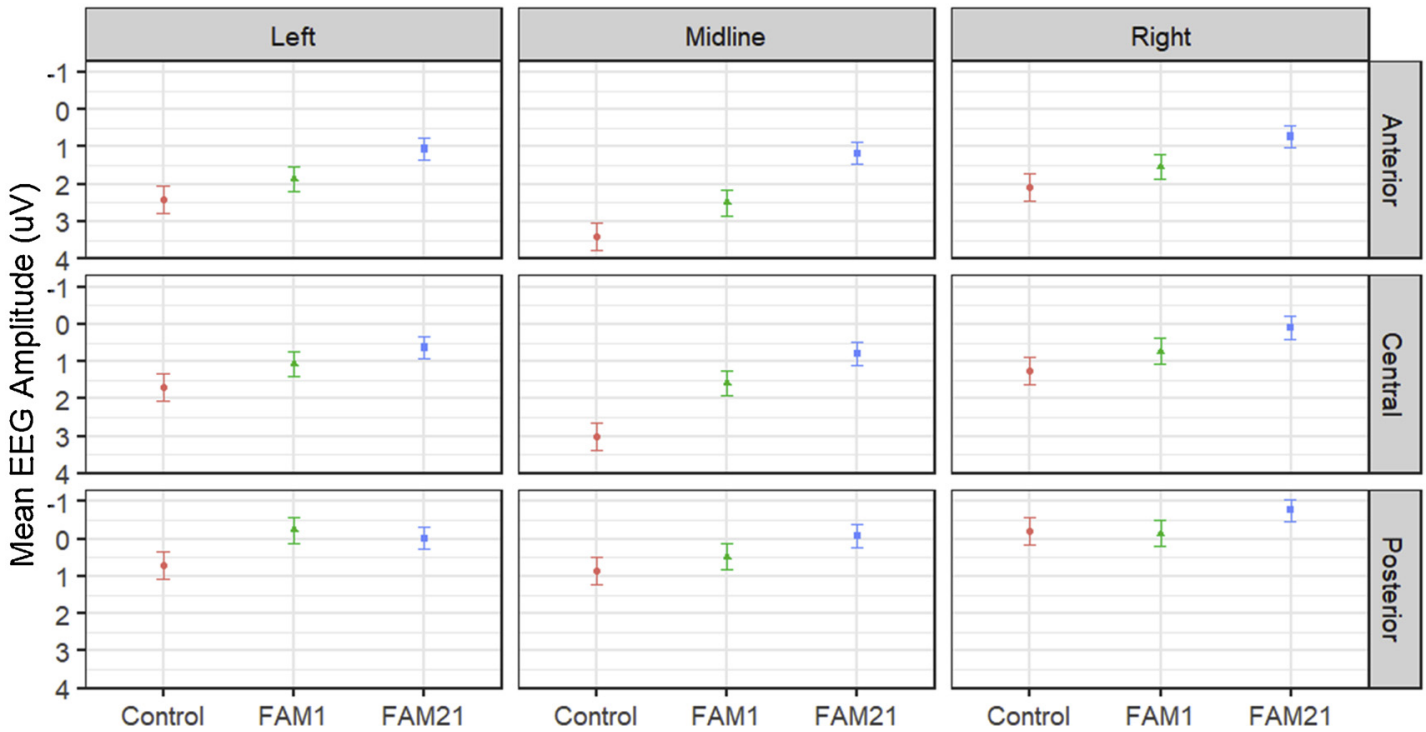

Fig. 6. A) The grand-average plots reflect selected electrodes that are representative for the regions of interest in the statistical model. The N2 component is highlighted by the blue band, for the time range of 160-240 ms and stimulus-locked to SRTT trial onset for all correct trials performed across Sequence Learning blocks. The FAM21 group completed 20 sessions of focused attention meditation (FAM) and a single FAM session immediately prior to the SRTT. FAM1 group completed 20 sessions of an audio-listening task (LT) and a single FAM session immediately prior to SRTT. The Control group completed 20 sessions of an LT and a single-session of LT immediately prior to the SRTT. B) Modelled effects for Sequence Learning N2 Amplitude: Group x Laterality x Sagittality. Error bars represent 95 $\%$ confidence intervals, they are small due to high consistency from correct responses. The results revealed that interaction effects were most prominent over anterior and central, but not posterior regions of interest.

significant contributions from the anterior mid cingulate cortex and the right inferior frontal cortex. In addition, the N2 is also considered an essential ERP component that is part of the performance monitoring network during the performance of goal-related tasks [92]. It is therefore possible in our case that cognitive control during sequence learning was supported by activation from the fronto-parietal network connected to posterior medial frontal cortex and dorsomedial prefrontal cortex $[55,67,93,94]$. The fronto-parietal network has been associated with the regulation of attention and working memory, and increased N2 activation is therefore suggestive of increased cognitive control supporting sequence learning performance [93,95]. Essentially, these areas are activated when speed and accuracy are prioritised as key goals for maintaining sequence performance $[55,94,96]$. Other studies have suggested that a single-session of FAM was enough to induce cognitive control effects [1,97] and in our case, the increased N2 amplitude from our single-session FAM group relative to Control, appears to support also this observation. In the next section, we will further expand on the understanding of FAM training on the dynamics of N2 and general 


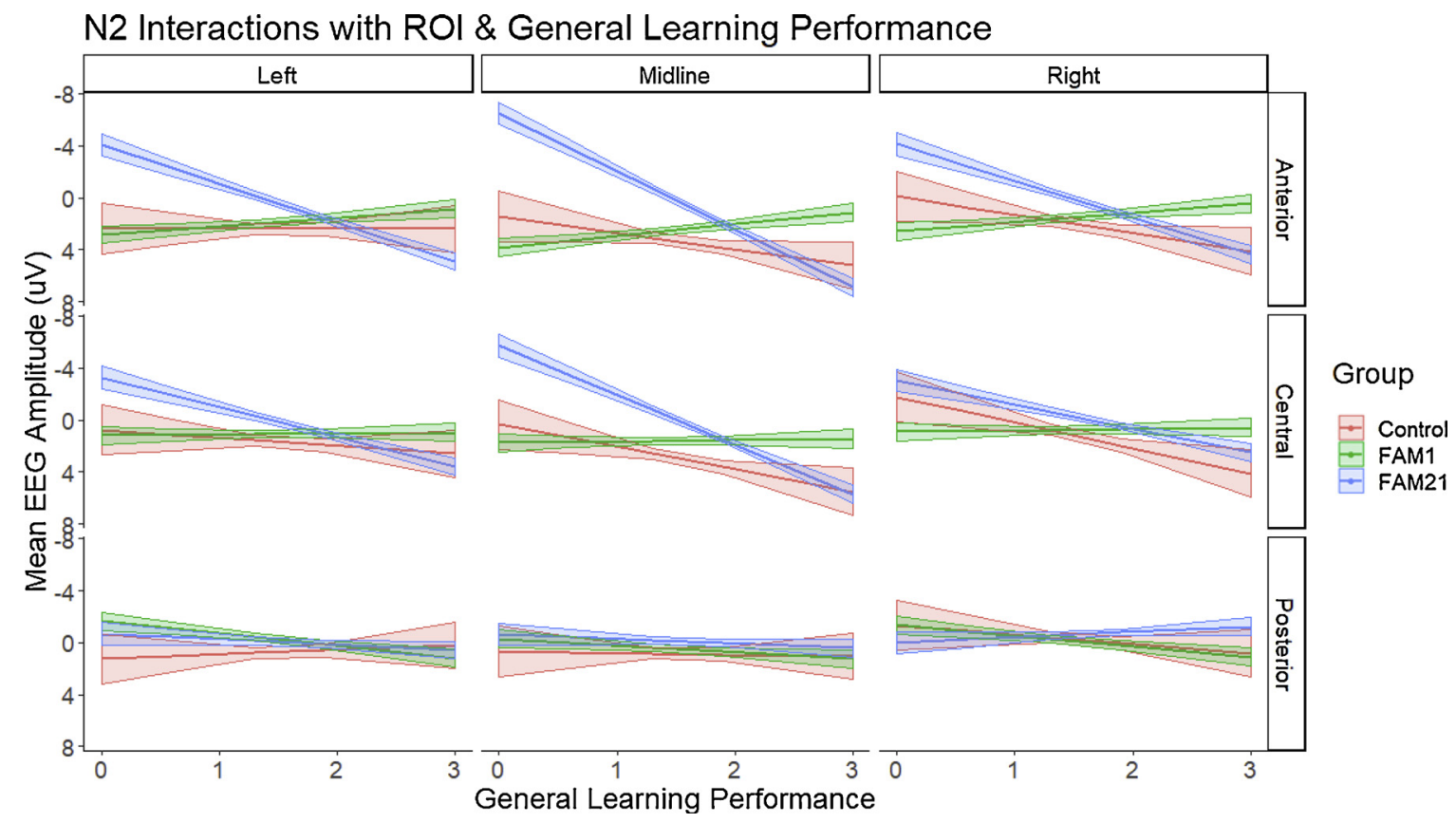

Fig. 7. Sequence Learning N2 Amplitude: Group x Laterality x Sagittality x General Learning Performance interactions. Modelled effects for the N2 stimulus-locked to SRTT trial onset for all correct trials performed during Sequence Learning blocks against General Learning Performance (raw beta coefficients organised into five levels of performance bins). The FAM21 group completed 20 sessions of focused attention meditation (FAM) and a single FAM session immediately prior to the SRTT. FAM1 group completed 20 sessions of an audio listening task (LT) and a single FAM session immediately prior to SRTT. The Control group completed 20 sessions of LT and a single-session of LT immediately prior to the SRTT. Filled colours represent $95 \%$ confidence intervals and are small due to high consistency from correct responses. Effects were most pronounced across anterior and central regions of interest (ROIs). The trends show that FAM21 had greater levels of top-down activation to support low levels of general learning performance compared to FAM1 and Control. As levels of general learning increased, FAM21 have greater decline of amplitudes compared to FAM1 and Control to support general learning performance.

\section{N2 Interactions with ROI \& Sequence-Specific Learning Performance}

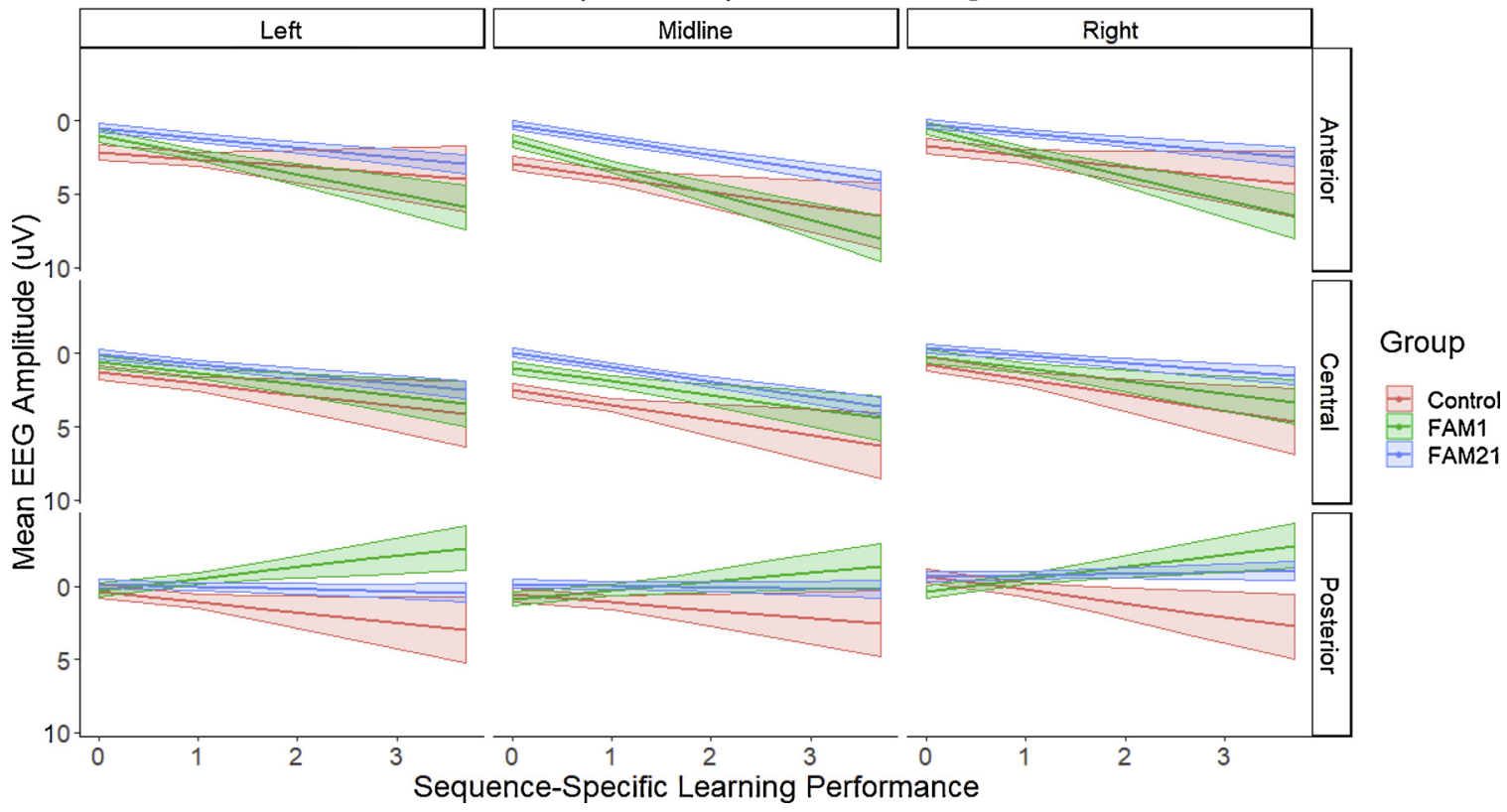

Fig. 8. Sequence Learning N2 Amplitude: Group x Laterality x Sagittality x Sequence-Specific Learning Performance interactions. Modelled effects for the N2 stimulus-locked to SRTT trial onset for all correct trials performed during Sequence Learning blocks against Sequence-Specific Performance (sequence-specific learning index organised into five levels of performance bins). The FAM21 group completed 20 sessions of focused attention meditation (FAM) and a single FAM session immediately prior to the SRTT. FAM1 group completed 20 sessions of an audio listening task (LT) and a single FAM session immediately prior to SRTT. The Control group completed 20 sessions of LT and a single-session of LT immediately prior to the SRTT. Filled colours represent $95 \%$ confidence intervals and very small due to high consistency from correct responses. The trends show that FAM21 had greater levels of top-down activation across the anterior regions compared to FAM1 across all levels of sequence-specific learning performance but not Control. As levels of sequence-specific learning increased, the central ROI regions showed all groups declined in their N2 amplitudes evenly. The posterior regions revealed that FAM21 maintained a consistent N2 amplitude whilst FAM1 increased and Control decreased in their amplitude, whilst sequence-specific performance increased. 
learning to understand mechanistic actions.

\subsection{Focused-attention meditation training effects on N2 dynamics, and general learning and sequence-specific performance}

To understand how behavioural performance correlated with N2 amplitudes, the present study utilised mixed effects models to understand general learning performance as a predictor for N2 amplitudes. By means of this approach, we addressed one of the limitations found in Moore et al. [11]. These results provide further evidence to support the assumption that $\mathrm{N} 2$ amplitude is sensitive to general learning performance in sequence learning $[15,68]$, and allow us to further extend the theoretical bases of cognitive control and stimulus-based strategies $[4,7,68,98]$. Firstly, we predicted that general-learning performance (that is closely related to stimulus-based strategy), would be most predictive of $\mathrm{N} 2$ amplitude changes. Indeed, our modelling clarifies this notion, with the general learning index showing the best fit for N2 amplitude changes as compared to sequence-specific learning. This evidence is in agreement with Daltrozzo \& Conway [15] and further reinforces support for the $\mathrm{N} 2$ as an index of cognitive control during sequence learning as compared to other ERP components such as the P300, that is believed to be related to memory and internal representation updates. Secondly, our results show that across the frontocentral areas exhibit the most prominent effects, and that FAM training supports a pattern of decreasing N2 amplitudes that is modulated by general learning performance.

The key interpretation is that FAM training affords much greater levels of cognitive control during lower levels of general learning in comparison to single-session FAM practise or LT control. At first glance our model results appear paradoxical considering that overall mean N2 amplitudes were higher across all Sequence Learning blocks, with significantly shorter behavioural RT outcomes with FAM training. Returning to the theoretical bases of the SRTT, which frequently reports that learning is typically predicated on block-by-block RT improvements [32,33]. However, if faster block RT performances were already demonstrated from block 1 (in our case), then throughout the course of sequence learning, there may be a possibility of reduced learning improvement (RT change differences across all learning trials). Faster initial RT performance would therefore appear to suppress general learning effect improvements, which could be misleading in trying to understand FAM training effects on sequence learning performances and N2 amplitude changes.

The modelling clarifies this with an additional layer of interpretation and shows that FAM training not only results in increased N2 amplitudes, but that the amplitudes do not stay stagnant throughout different indices of sequence learning. Both single-session FAM and performing a listening control task appears to result in less dynamic changes, with either increases or decreases in amplitudes across the scalp ROIs. However, FAM training appears to support a consistent decrease in N2 amplitudes across the frontocentral ROIs as general learning performance increases. Reductions in amplitudes during high level motor performances are essentially evidences for neural efficiencies in the cognitive system leading to expertise and increased predictability of future sequence learning performances [99-101]. Our results suggests that following FAM training, and if one shows high levels of general learning performance during sequence learning, less neural activation can be expected to support that high level of performance, and therefore suggestive of greater efficiencies arising [15].

Moving onto cognitive control and sequence-specific learning performance, the model revealed that all groups were performing similarly in the behavioural aspects, and even when modelled with N2 amplitudes, interactions were not evident. This indicates that FAM training does not increase/decrease but maintains on par performance of sequence-specific learning when compared with performing other cognitive tasks' conditions. The model also reveals that across all the groups, N2 amplitudes reduced in a similar manner when sequence- specific performance improved with less dramatic decreases in comparison to general learning performance. The evidence shown here in combination with better model fits further support that general learning performance is the main form of performance enhancement and not sequence-specific learning.

General learning performance, also referred as perceptual learning [15], is one of the essential components of sequence learning and acts as a precursor to sequence-specific learning. It is believed that general learning facilitates the development of spatio-temporal representations using shared neural networks with sequence-specific learning [102], but more importantly can also be modulated by attention and awareness $[15,103,104]$. Our results point towards the possibility that participants in the FAM21 group, due to FAM training, had developed increased vigilance towards upcoming stimuli by pre-activating cognitive control resources (including attention). This facilitated faster responding by being already 'prepared' for responding. In terms of FAM cyclical stages, it is equivalent to the 'focus on object'/ 'sustaining of attention' and maintenance of the 'Alerting' network stage (Graphical abstract). Hence, N2 amplitudes in the anterior-central ROIs were elevated on an overall mean level and also when modelled against general learning performance. This increased activation eventually starts to become more efficient at later stages when learners start to build their internal representation of the sequence [5,24]. Equal performance across groups in sequence-specific learning show that such increased N2 amplitudes do not hamper the development of internal representation of the sequence. The next part further explains the possible mechanismi that transfers from FAM training to sequence learning.

FAM training represents a cognitive skill that can be developed with experience like many other skills to reach increased levels of expertise [51]. The goal of FAM is to improve attentional control but because of its cyclical nature of switching between distraction and object focus during practise, a general set of cognitive control processes shared by other goal-orientated tasks are also utilised [10,52]. Over time, with regular practice, it is plausible that effects gained in meditation may also transfer to other tasks that activate the same cognitive control processes/ brain networks. Relatively brief training durations such as 4 days [105] have shown that meditation can alter brain activation patterns during practice with reinforced attentional effects. Consequently, our training duration was also expected to exert effects. Specific to the sequence learning, we propose that increased FAM experience/expertise results in establishing increased cognitive control states with stronger transfer and efficiencies during learning. FAM training also appears to facilitate reduced cognitive control activation during higher levels of general learning effects compared to single-session FAM and LT Control. Specifically, with the patterns observed in N2 amplitudes across both general and sequence-specific learning, we interpret that FAM expertise allows for learners to maintain a reinforced and focussed vigilance when performing sequence learning, when attending to stimuli targets.

In conclusion, additional benefit from FAM training can be expected in support of greater use of stimulus-based control strategies [1,3,7] and more effective dynamic modulation of cognitive control: increased cognitive control during low levels of general learning performance and becoming more efficient (less N2 amplitudes and faster performances) at higher levels in overall sequence learning performance.

\subsection{Methodological strengths, limitations and future directions}

Participants in the current study were naïve and blinded to the experimental aims, with the goal to limit potential influences associated with the expectations of the beneficial or negative effects of meditation [77]. Another strength was the inclusion of an active control condition in the form of a listening control task. The period of sequence training was relatively short (one session of 14 blocks) and therefore the influence of meditation across several sessions of learning may be different (multi-days with different second-order conditional sequences) [34] 
which may provide insight to temporal effects. Testing multiple days of sequence learning would also overcome the issue of using pseudorandom blocks in familiarisation for better comparisons of pre-post effects, although this would greatly increase participant burden. The transfer block in our experiment was also designed as random sequence presentation - for future work, a different SOC would have been a better design to maintain a structural comparison between the old SOC in the last learning block and the transfer block. Another approach may be to use a more procedural sequence learning task such as the Discrete Sequence Task (DSP) [106], in that general and sequence-specific effects can be more clearly separated and modelled with ERP/EEG changes. In addition, when considering only sequence learning without cognitive control manipulations, further questions surrounding other ERP components such as the P300, N400 and P600 changes in amplitudes could be revealed [15,84].

With regard to FAM, it is unknown if cognitive control effects require reinstatement prior to learning and/or whether varying FAM duration may produce different effects. The insertion of FAM practice in-between blocks has also not been investigated, which may further strengthen cognitive control between block performances. Other factors such as personal predispositions, meditation styles, duration of practice and temporal placement should also be considered to further understand meditation effects and/or sequence learning effects independently. The inclusion of other neuropsychological tests such as The Philadelphia Mindfulness Scale [107], Stroop Test [108] or The D2 Test of Attention [109] may provide insight when modelled against sequence learning outcomes and ERPs.

Lastly, because we have shown that attentional and cognitive control states can be biased, it would also be valuable to investigate the reversed effect of sequence learning for meditation. This may show that effects from various preceding task may also influence the quality of meditation practice to highlight inert effects in cognitive control states. These are all important directions that aim to further understand the mechanistic actions by which cognitive control states enhance motor learning or other cognitive task performances.

\subsection{Summary: Training and instantaneous forms of focused-attention meditation enhancement of cognitive control during sequential behaviour}

This was the first study that aimed to bridge key areas of sequence learning theory by investigating if electrophysiological correlates of cognitive control could be enhanced following single-session FAM, and by FAM training. Firstly, our results support and replicated the finding that single-session FAM can increase cognitive control, as indexed by N2 amplitude, and modulate stimulus-based control as well as enhance general learning effects. The results also supported our predictions that FAM training further enhanced single-session FAM effects with greater levels of cognitive control reflected in a further increase of N2 amplitude. The modelling of N2 amplitudes against different behavioural performance predictors suggested that general learning effects provided the best fit. More importantly, our results revealed that, with FAM training, one can expect the greatest rate of decline in N2 amplitudes from initial to higher levels of general learning performances, indicating a dynamic modulation of cognitive control during sequence learning as a neurobiologically plausible mechanistic action.

\section{Acknowledgements}

We thank David Burgess for providing a recording of the yoga nidra meditation used in this experiment. We also express gratitude to the lab researchers and research assistants that helped to collect data and further assistance in this study: Alex Chatburn, Andrew Corcoran, Christina Lubinus, Erica Wilkinson, Schae Matthews, Matthew Seals and Dmitry Altukhov. This research was supported by the Australian Government Research Training Program Scholarship, and the Russian Science Foundation project (RSF-19-18-00534) for Russell W. Chan. Ina
Bornkessel-Schlesewsky acknowledges the support of an Australian Research Council Future Fellowship (FT160100437).

\section{Appendix A. Supplementary data}

Supplementary material related to this article can be found, in the online version, at doi:https://doi.org/10.1016/j.bbr.2020.112536.

\section{References}

[1] M.A. Immink, et al., Sequence Learning Enhancement Following Single-Session Meditation Is Dependent on Metacontrol Mode and Experienced Effort, J. Cogn Enhanc. 1 (2) (2017) 127-140.

[2] E.L. Abrahamse, et al., Control of automated behavior: insights from the discrete sequence production task, Front. Hum. Neurosci. 7 (2013) 82.

[3] R.W. Chan, K. Lushington, M.A. Immink, States of focused attention and sequential action: A comparison of single session meditation and computerised attention task influences on top-down control during sequence learning, Acta Psychol. (Amst) 191 (2018) 87-100.

[4] E. Tubau, B. Hommel, J. Lopez-Moliner, Modes of executive control in sequence learning: from stimulus-based to plan-based control, J. Exp. Psychol. Gen. 136 (1) (2007) 43-63.

[5] W.B. Verwey, C.H. Shea, D.L. Wright, A cognitive framework for explaining serial processing and sequence execution strategies, Psychon. Bull. Rev. 22 (1) (2015) 54-77.

[6] E. Abrahamse, et al., Grounding cognitive control in associative learning, Psychol. Bull. 142 (7) (2016) 693-728.

[7] R.W. Chan, M.A. Immink, K. Lushington, The influence of focused-attention meditation states on the cognitive control of sequence learning, Conscious. Cogn. 55 (2017) 11-25.

[8] B.R. Cahn, J. Polich, Meditation states and traits: EEG, ERP, and neuroimaging studies, Psychol. Bull. 132 (2) (2006) 180-211.

[9] M.J. Larson, P.R. Steffen, M. Primosch, The impact of a brief mindfulness meditation intervention on cognitive control and error-related performance monitoring, Front. Hum. Neurosci. 7 (2013) 308.

[10] P. Malinowski, Neural mechanisms of attentional control in mindfulness meditation, Front. Neurosci. 7 (2013) 8.

[11] A. Moore, et al., Regular, brief mindfulness meditation practice improves electrophysiological markers of attentional control, Front. Hum. Neurosci. 6 (2012) 18.

[12] M.J. Nissen, P. Bullemer, Attentional requirements of learning: evidence from performance measures, Cogn. Psychol. 19 (1) (1987) 1-32.

[13] B.A. Clegg, G.J. Digirolamo, S.W. Keele, Sequence learning, Trends Cogn Sci 2 (8) (1998) 275-281.

[14] S.W. Keele, et al., The cognitive and neural architecture of sequence representation, Psychol. Rev. 110 (2) (2003) 316-339.

[15] J. Daltrozzo, C.M. Conway, Neurocognitive mechanisms of statistical-sequential learning: what do event-related potentials tell us? Front. Hum. Neurosci. 8 (2014) 437.

[16] E.L. Abrahamse, et al., Representing serial action and perception, Psychon. Bull. Rev. 17 (5) (2010) 603-623.

[17] B. Hommel, The prepared reflex: automaticity and control in stimulus-response translation, in: S. Monsell, J. Driver (Eds.), Control of Cognitive Processes: Attention and Performance XVIII, MIT Press, Cambridge, MA, 2000, p. 26.

[18] A. Franco, A. Destrebecqz, Chunking or not chunking? How do we find words in artificial language learning? Adv. Cogn. Psychol. 8 (2) (2012) 144-154.

[19] W.B. Verwey, D.L. Wright, Learning a keying sequence you never executed: evidence for independent associative and motor chunk learning, Acta Psychol. (Amst) 151 (2014) 24-31.

[20] L. Jimenez, Taking patterns for chunks: is there any evidence of chunk learning in continuous serial reaction-time tasks? Psychol. Res. 72 (4) (2008) 387-396.

[21] L. Jimenez, Attention and Implicit Learning, John Benjamins Pub., 2003.

[22] G. Borragan, et al., Cognitive fatigue facilitates procedural sequence learning, Front. Hum. Neurosci. 10 (2016) 86.

[23] T. Amer, K.L. Campbell, L. Hasher, Cognitive Control As a Double-Edged Sword, Trends Cogn Sci 20 (12) (2016) 905-915.

[24] M.A. Immink, W.B. Verwey, D.L. Wright, The neural basis of cognitive efficiency in motor skill performance across early learning to automatic stages, in: S. Nam (Ed.), Neuroergonomics: Principles and Practice, ed., Springer, 2019.

[25] A. Miyake, et al., The unity and diversity of executive functions and their contributions to complex "Frontal Lobe" tasks: a latent variable analysis, Cogn. Psychol. 41 (1) (2000) 49-100.

[26] G. Gratton, P. Cooper, M. Fabiani, C.S. Carter, F. Karayanidis, Dynamics of cognitive control: theoretical bases, paradigms, and a view for the future, Psychophysiology 55 (2018) e13016, https://doi.org/10.1111/psyp.13016 https://onlinelibrary.wiley.com/action/showCitFormats?doi=10.1111\%2Fpsyp. 13016.

[27] W.B. Verwey, B.A. Clegg, Effector dependent sequence learning in the serial RT task, Psychol. Res. 69 (4) (2005) 242-251.

[28] S.E. Petersen, M.I. Posner, The attention system of the human brain: 20 years after, Annu. Rev. Neurosci. 35 (2012) 73-89.

[29] M.I. Posner, S.E. Petersen, The attention system of the human brain, Annu. Rev. Neurosci. 13 (1990) 25-42. 
[30] A. Miyake, N.P. Friedman, The Nature and Organization of Individual Differences in Executive Functions: Four General Conclusions, Curr. Dir. Psychol. Sci. 21 (1) (2012) 8-14.

[31] E.L. Abrahamse, M.L. Noordzij, Designing training programs for perceptual-motor skills: practical implications from the serial reaction time task, Rev. Eur. Psychol. Appliquã(C)/European Rev. Appl. Psychol. 61 (2) (2011) 65-76.

[32] E.M. Robertson, The serial reaction time task: implicit motor skill learning? J. Neurosci. 27 (38) (2007) 10073-10075.

[33] H. Schwarb, E.H. Schumacher, Generalized lessons about sequence learning from the study of the serial reaction time task, Adv. Cogn. Psychol. 8 (2) (2012) $165-178$.

[34] T. Verstynen, et al., Dynamic sensorimotor planning during long-term sequence learning: the role of variability, response chunking and planning errors, PLoS One 7 (10) (2012) e47336.

[35] P. Chiappe, L. Hasher, L.S. Siegel, Working memory, inhibitory control, and reading disability, Mem. Cognit. 28 (1) (2000) 8-17.

[36] M.J. Kane, R.W. Engle, Working-memory capacity, proactive interference, and divided attention: limits on long-term memory retrieval, J. Exp. Psychol. Learn. Mem. Cogn. 26 (2) (2000) 336-358.

[37] J.A. Alvarez, E. Emory, Executive function and the frontal lobes: a meta-analytic review, Neuropsychol. Rev. 16 (1) (2006) 17-42.

[38] O.E. Krigolson, et al., Learning to become an expert: reinforcement learning and the acquisition of perceptual expertise, J. Cogn. Neurosci. 21 (9) (2009) 1834-1841.

[39] T. Amer, L. Hasher, Conceptual processing of distractors by older but not younger adults, Psychol. Sci. 25 (12) (2014) 2252-2258.

[40] B. Hommel, Between persistence and flexibility, in: A.J. Elliot (Ed.), The Yin and Yang of Action Control, in Advances in Motivation Science, Elsevier, 2015, pp. 33-67.

[41] C. Lustig, L. Hasher, S.T. Tonev, Distraction as a determinant of processing speed, Psychon. Bull. Rev. 13 (4) (2006) 619-625.

[42] J.F. Schwab, et al., Aging and the statistical learning of grammatical form classes, Psychol. Aging 31 (5) (2016) 481-487.

[43] B. Hommel, L.S. Colzato, Meditation and Metacontrol, J. Cogn. Enhanc. 1 (2) (2017) 115-121.

[44] A.D. Allport, E.A. Styles, S. Hsieh, Shifting Attentional Set - Exploring the Dynamic Control of Tasks, Vol. Vol. XV. (1994).

[45] Y.N. Delevoye-Turrell, C. Bobineau, Motor consciousness during intention-based and stimulus-based actions: modulating attention resources through mindfulness meditation, Front. Psychol. 3 (2012) 290.

[46] D. Nemeth, K. Janacsek, J. Fiser, Age-dependent and coordinated shift in performance between implicit and explicit skill learning, Front. Comput. Neurosci. 7 (2013) 147.

[47] Y.Y. Tang, B.K. Holzel, M.I. Posner, The neuroscience of mindfulness meditation, Nat. Rev. Neurosci. 16 (4) (2015) 213-225.

[48] A.P. Jha, J. Krompinger, M.J. Baime, Mindfulness training modifies subsystems of attention, Cogn. Affect. Behav. Neurosci. 7 (2) (2007) 109-119.

[49] A. Lutz, et al., Investigating the phenomenological matrix of mindfulness-related practices from a neurocognitive perspective, Am. Psychol. 70 (7) (2015) 632-658.

[50] H.A. Slagter, R.J. Davidson, A. Lutz, Mental training as a tool in the neuroscientific study of brain and cognitive plasticity, Front. Hum. Neurosci. 5 (2011) 17.

[51] S.N. Gallant, Mindfulness meditation practice and executive functioning: breaking down the benefit, Conscious. Cogn. 40 (2016) 116-130.

[52] W. Hasenkamp, L.W. Barsalou, Effects of meditation experience on functional connectivity of distributed brain networks, Front. Hum. Neurosci. 6 (2012) 38.

[53] N.G. Müller, R.T. Knight, Age-related changes in fronto-parietal networks during spatial memory: an ERP study, Cogn. Brain Res. 13 (2) (2002) 221-234.

[54] S.J. Luck, G.F. Woodman, E.K. Vogel, Event-related potential studies of attention, Trends Cogn Sci 4 (11) (2000) 432-440.

[55] M. Ullsperger, et al., Neural mechanisms and temporal dynamics of performance monitoring, Trends Cogn Sci 18 (5) (2014) 259-267.

[56] J.R. Folstein, C. Van Petten, Influence of cognitive control and mismatch on the N2 component of the ERP: a review, Psychophysiology 45 (1) (2008) 152-170.

[57] W. Ritter, R. Simson, H.G. Vaughan Jr., Event-related potential correlates of two stages of information processing in physical and semantic discrimination tasks, Psychophysiology 20 (2) (1983) 168-179.

[58] J.H. Satterfield, et al., Ontogeny of selective attention effects on event-related potentials in attention-deficit hyperactivity disorder and normal boys, Biol. Psychiatry 28 (10) (1990) 879-903.

[59] W.C. McCallum, Some sensory and cognitive aspects of ERPs: a review, in: H.H. Kornhubek, L. Deecke (Eds.), Progress in Brain Research, Elsevier, 1980, pp. 261-278.

[60] E. Donchin, W. Ritter, C. McCallum, Cognitive psychophysiology: the endogenous components of the ERP, in: E. Callaway, P. Tueting, S. Koslow (Eds.), Brain EventRelated Potentials in Man. Academic Press, New York, 1978.

[61] B.I. Jack, et al., The Event-Related Brain Potential as an Index of DisplayMonitoring Workload, Hum. Factors 22 (2) (1980) 211-224.

[62] K.B. Baldwin, M. Kutas, An ERP analysis of implicit structured sequence learning, Psychophysiology 34 (1) (1997) 74-86.

[63] M. Eimer, et al., Explicit and implicit learning of event sequences: evidence from event-related brain potentials, J. Exp. Psychol. Learn. Mem. Cogn. 22 (4) (1996) 970-987.

[64] N.K. Ferdinand, et al., Event-related potential correlates of declarative and nondeclarative sequence knowledge, Neuropsychologia 48 (9) (2010) 2665-2674.

[65] J. Russeler, F. Rosler, Implicit and explicit learning of event sequences: evidence for distinct coding of perceptual and motor representations, Acta Psychol. (Amst)
104 (1) (2000) 45-67.

[66] J. Russeler, D. Kuhlicke, T.F. Munte, Human error monitoring during implicit and explicit learning of a sensorimotor sequence, Neurosci. Res. 47 (2) (2003) 233-240.

[67] C. Danielmeier, et al., Posterior medial frontal cortex activity predicts post-error adaptations in task-related visual and motor areas, J. Neurosci. 31 (5) (2011) $1780-1789$.

[68] A. Kobor, et al., ERPs differentiate the sensitivity to statistical probabilities and the learning of sequential structures during procedural learning, Biol. Psychol. 135 (2018) 180-193.

[69] W.H. Alexander, J.W. Brown, Computational models of performance monitoring and cognitive control, Top. Cogn. Sci. 2 (4) (2010) 658-677.

[70] C.D. Wickens, et al., Effectiveness of part-task training and increasing-difficulty training strategies: a meta-analysis approach, Hum. Factors 55 (2) (2013) 461-470.

[71] E. Tubau, et al., Individual differences in sequence learning and auditory pattern sensitivity as revealed with evoked potentials, Eur. J. Neurosci. 26 (1) (2007) 261-264.

[72] S. van Leeuwen, W. Singer, L. Melloni, Meditation increases the depth of information processing and improves the allocation of attention in space, Front. Hum. Neurosci. 6 (2012) 133.

[73] J. Reed, P. Johnson, Assessing implicit learning with indirect tests: determining what is learned about sequence structure, J. Exp. Psychol. Learn. Mem. Cogn. 20 (3) (1994) 9.

[74] J.D. Nash, A. Newberg, Toward a unifying taxonomy and definition for meditation, Front. Psychol. 4 (2013) 806.

[75] S. Saraswati, Yoga Nidra, Yoga Publications Trust, 2002.

[76] W. Hasenkamp, et al., Mind wandering and attention during focused meditation: a fine-grained temporal analysis of fluctuating cognitive states, Neuroimage 59 (1) (2012) 750-760.

[77] R.J. Davidson, A.W. Kaszniak, Conceptual and methodological issues in research on mindfulness and meditation, Am. Psychol. 70 (7) (2015) 581-592.

[78] E.E. Rexford, Home Floriculture; a Practical Guide to the Treatment of Flowering and Other Ornamental Plants in the House and Garden, New York Orange Judd company., 1916

[79] A. Gramfort, et al., MEG and EEG data analysis with MNE-Python, Front. Neurosci. 7 (267) (2013)

[80] D. Bates, et al., Fitting Linear Mixed-Effects Models Using lme4, Statistical software 67 (1) (2015) 48

[81] R. Team, R Studio: Integrated Development for R, R Studio, Inc., Boston, MA, 2015.

[82] K. Oberauer, R. Kliegl, A formal model of capacity limits in working memory, J. Mem. Lang. 55 (4) (2006) 601-626.

[83] R. Lenth, et al., Emmeans: Estimated Marginal Means, Aka Least-squares Means, (2019) https://cran.r-project.org/web/packages/emmeans/index.html.

[84] C. Beaulieu, et al., Electrophysiological correlates of motor sequence learning, BMC Neurosci. 15 (1) (2014) 102.

[85] B.R. Burnham, M. Sabia, C. Langan, Components of working memory and visual selective attention, J. Exp. Psychol. Hum. Percept. Perform. 40 (1) (2014) 391-403.

[86] J. Fox, S. Weisberg, Visualizing fit and lack of fit in complex regression models with predictor effect plots and partial residuals, J. Stat. Softw. 87 (9) (2018) 26.

[87] S. Song, J.H. Howard Jr., D.V. Howard, Perceptual sequence learning in a serial reaction time task, Exp. Brain Res. 189 (2) (2008) 145-158.

[88] R.T. Knight, Evoked Potential Studies of Attention Capacity in Human Frontal Lobe Lesions, in Frontal Lobe Function and Dysfunction, Oxford University Press, New York, NY, US, 1991, pp. 139-153.

[89] J. Polich, Updating P300: an integrative theory of P3a and P3b, Clin. Neurophysiol. 118 (10) (2007) 2128-2148.

[90] B. Hommel, et al., The Theory of Event Coding (TEC): a framework for perception and action planning, Behav. Brain Sci. 24 (5) (2001) 849-878 discussion 878-937.

[91] T.O.J. Gruendler, M. Ullsperger, R.J. Huster, Event-related potential correlates of performance-monitoring in a lateralized time-estimation task, PLoS One 6 (10) (2011) e25591.

[92] M. Ullsperger, C. Danielmeier, G. Jocham, Neurophysiology of performance monitoring and adaptive behavior, Physiol. Rev. 94 (1) (2014) 35-79.

[93] D.T. Stuss, R.T. Knight, Principles of Frontal Lobe Function, OUP USA., 2013.

[94] E.H. Schumacher, M.J. Hendricks, M. D’Esposito, Sustained involvement of a frontal-parietal network for spatial response selection with practice of a spatial choice-reaction task, Neuropsychologia 43 (10) (2005) 1444-1455.

[95] E. Koechlin, C. Ody, F. Kouneiher, The architecture of cognitive control in the human prefrontal cortex, Science 302 (5648) (2003) 1181-1185.

[96] J.R. Wessel, et al., Lesions to the prefrontal performance-monitoring network disrupt neural processing and adaptive behaviors after both errors and novelty, Cortex 50 (2014) 45-54.

[97] L.S. Colzato, et al., A single bout of meditation biases cognitive control but not attentional focusing: evidence from the global-local task, Conscious. Cogn. 39 (2016) 1-7.

[98] W.B. Verwey, E.L. Abrahamse, Distinct modes of executing movement sequences: reacting, associating, and chunking, Acta Psychol. (Amst) 140 (3) (2012) 274-282.

[99] J. Fan, et al., Testing the efficiency and independence of attentional networks, J. Cogn. Neurosci. 14 (3) (2002) 340-347.

[100] T.L. Ansari, N. Derakshan, The neural correlates of cognitive effort in anxiety: effects on processing efficiency, Biol. Psychol. 86 (3) (2011) 337-348.

[101] K. Friston, et al., Active inference: a process theory, Neural Comput. 29 (1) (2017) $1-49$. 
[102] N.B. Turk-Browne, et al., Neural evidence of statistical learning: efficient detection of visual regularities without awareness, J. Cogn. Neurosci. 21 (10) (2009) 1934-1945.

[103] R.L. Goldstone, Perceptual learning, Annu. Rev. Psychol. 49 (1998) 585-612.

[104] Y. Sasaki, J.E. Nanez, T. Watanabe, Advances in visual perceptual learning and plasticity, Nat. Rev. Neurosci. 11 (1) (2010) 53-60.

[105] F. Zeidan, et al., Neural correlates of mindfulness meditation-related anxiety relief, Soc. Cogn. Affect. Neurosci. 9 (6) (2014) 751-759.
[106] W.B. Verwey, Evidence for a multistage model of practice in a sequential movement task, J. Exp. Psychol. Hum. Percept. Perform. 25 (6) (1999) 1693-1708.

[107] L. Cardaciotto, et al., The assessment of present-moment awareness and acceptance: the Philadelphia Mindfulness Scale, Assessment 15 (2) (2008) 204-223.

[108] J.R. Stroop, Studies of interference in serial verbal reactions, J. Exp. Psychol. 18 (6) (1935).

[109] R.M. Ross, The D2 Test of Attention: An Examination of Age, Gender, and Crosscultural Indices, Argosy University, 2005. 